\title{
MICROBIOREACTORS AS ENGINEERING TOOLS FOR BIOPROCESS DEVELOPMENT
}

\author{
R. C. Prado ${ }^{1 *}$ and E. R. Borges ${ }^{1}$ \\ ${ }^{1}$ Department of Biochemical Engineering, School of Chemistry, Federal University of Rio de Janeiro, Avenida Athos da Silveira Ramos, \\ 21941-909 - Rio de Janeiro, Brazil.
}

(Submitted: August 14, 2017 ; Revised: February 2, 2018 ; Accepted: February 26, 2018)

\begin{abstract}
Within the engineering and development of industrial bioprocesses, microbioreactors are microfluidic tools able to provide high-throughput screening tests in a fast and cheap manner by using small amounts of reagents. In addition, such tools are versatile and may allow a better controllability of parameters when compared to conventional bench-scale reactors. Consequentially, this technology has been gaining attention from the scientific community over the past years. In such scenario, this work provides a review study of the microbioreactor technology, outlining the origin, main concepts and principles of such technology, aiming to elucidate general questions that may emerge when studying such an approach. Past and current approaches are discussed aiming at drawing a comparative picture about such technology regarding future developments.

Keywords: Microbioreactor; Microfluidics; Industrial biotechnology; Bioprocess; Fermentation.
\end{abstract}

\section{INTRODUCTION}

Over the past decades, the miniaturization of bioreactors has been intensively studied with a perspective of great new high-throughput techniques for biochemical approaches (Whitesides, 2006). Deriving from the early researches on microfluidics, a science that studies and manipulates small amounts of fluid, microbioreactors compose a technology that provides the possibility of carrying out bioprocesses under the micro scale. By combining both microfluidics and biochemical principles, such scale down thus brings advantages over the traditional method that uses bench-scale bioreactors as it enables carrying out bioprocess experiments under dynamical and flexible conditions (Szita et al., 2005; Micheletti e Lye, 2006; Schäpper et al., 2009). Therefore, such a strategy can allow high-throughput experimental data via cheap and precise methods (Schäpper et al., 2009).

Within the industrial field, several variables are found to act as bottlenecks for the improvement of many bioprocesses due to their high complexity and relatively poor information regarding their nature (Schmidell et al., 2001; Micheletti and Lye, 2006). As in academia, many bioprocesses are not taken forward because their production rates are not feasible for an industrial scale or simply because of the lack of knowledge on specific variables (Schmidell et al., 2001). In both cases, the application of the microbioreactor technology would make a complete study of such variables possible, offering enough understanding of their behaviour and thus overcoming such bottlenecks and opening a new pathway for further optimizations.

The benefits offered by the application of microbioreactors to bioprocesses are due to three main differential advantages that have great impact on the achievement of relevant data. The first differential may be the simples and most obvious feature of such technology: the tiny amount of reagent required to carry out experimentations. Because microfluidics is based on the micro scale, the volumes of media, inoculum and other additional reagents applied in a microbioreactor are substantially small, influencing directly on the outlay of experimentation assays

\footnotetext{
* Correspondence: Mr. Raphael C Prado, E-mail raphael.prado@tum.de
} 
(Whitesides, 2006). The second differential consists in the time spent for the achievement of data. Given the small working volume required by this technology, less molecules are submitted to bio- and chemical transformations, reducing the time spent until the final step of a bioprocess (Micheletti and Lye, 2006). This is a relevant point to be considered when several assays need to be carried out. For example, optimizing bioprocesses can be very time-consuming when executing assays on bench scale while every single relevant variable is tested. The parallelization (scaling out) of such assays is restricted by the availability of facilities limiting several research centres to a singular execution of the experiments. On the other hand, several microbioreactors can run in parallel (Szita et al., 2005), reducing the expenses of experimentation as fewer facilities are required and more data is produced in less time. The third differential is a consequence of the microfabrication technology: there is a substantial reduction of human handling of bioprocesses when using microbioreactors since there is the possibility of automation for both upstream and downstream steps while using such technology. This feature has an effect on the superiority of resolution and precision of data, allowing a better reproducibility and standardization of the bioprocess itself (Szita et al., 2005; Schäpper et al., 2010; Hegab et al., 2013). Therefore, a minor experimental error is expected when using the microbioreactor technology, granting a higher level of reliability of results and assumptions.

Such advantages exert great influence on the strategies for researching the most diverse topics of bioprocesses since there is a substantial reduction in price and time required, allied with an increase in the precision of data (Szita et al., 2005; Micheletti and Lye, 2006; Schäpper et al., 2009; Hegab et al., 2013). Microbioreactors can thus comprehend a great alternative for companies and research centres that have to deal with limitations in financial support and facilities.

Due to the versatility of such devices, as long as microbioreactors were being realized, different nature, sizes and shapes were being adopted in such technology. As a consequence, the main concept of microbioreactor has varied from work to work, thus making necessary the restriction of the general features of such devices in several studies. As discussed later on, there are two current approaches concerning microbioreactors: microwell bioreactors and microfluidic bioreactors. Aiming to avoid a misunderstanding over the reading, when microwell bioreactors are not specified, this work will consider microbioreactors to be microfluidic bioreactor devices, concerning the high versatility and thus greater application of such approach.

In regard to the advent of microfabrication and microtechnology, the present review will discuss the various approaches of microfluidics with a particular focus on microbioreactors and their application to bioprocesses. Therefore, with the aim of introducing and substantiating the advent of microbioreactors, this review commences with a brief history about the emergence of miniaturized bioreactors, followed by a brief overview of the current scenario of published works concerning microbioreactors. In sequence, the second part of this work presents an overview about microfabrication and the principal materials and methods adopted for the establishment of a general device. As microbioreactors are only one of the multiple applications that microfluidics can provide, some of the popular approaches of microfluidics are also presented. Finally, the third part of this work will focus on miniaturized bioreactors and the key features necessary to establish an effective fermentation, going through heat and mass transfer, on-line monitoring of parameters and integration of microbioreactor instrumentation.

\section{THE ORIGINS OF MICROBIOREACTORS}

The emergence of microbioreactors is a derivate pathway from the association of two main fields: microfluidics technology and molecular biology. At the beginning of the 1950 decade, the development of photolithography gave birth to microfabrication, allowing scientists to develop the first microdevices: the micro-sized transistors.

As microtechnology gained new approaches and applications through the years, chemical assays commenced to be miniaturized. Consequently, Terry et al. (1979) published the very first miniaturized gasphase chromatography (GPC) device, a prototype almost three orders of magnitude smaller than conventional laboratory scales. Since then, it did not take long for engineers to sophisticate other microanalytical methods (such as high-performance liquid chromatography (HPLC) and capillary electrophoresis (CE)), increasing dramatically the capabilities of chemical analyses by relying on the capillary format (Whitesides, 2006). The emergence of these techniques allowed the obtainment of highprecision data with a high level of resolution by using small amounts of samples. This concept was the first motivation for the development of microanalytical strategies, which came out during the 1980s with a second motivation: the revolution of molecular biology by genomics. The technology was rapidly applied to biological purposes because it could provide the high levels of throughput and precision required by genomics (Whitesides, 2006). As a consequence, microchips were then developed based on polymers, an adaption of microfabrication techniques which came to be called soft-lithography. 
In the early 1990 s scientists commenced to explore the possibilities of compartmentalization and integration of miniaturized biochemical assays. The main idea consisted in developing devices able to host different biochemical assays in integration, resuming a whole laboratory into a small chip. The socalled Lab-on-a-chip technology then emerged as long as the advances in instrumentation would allow it. Interested in the versatility of such technology, several military programmes provided financial funding for the development of devices able to counter biological and chemical weapons. This came particularly from the Defence Advanced Research Projects Agency (DARPA), from the Department of Defence of The United States of America, resulting in a great stimulus for the development of microfluidic devices in the academic environment. Because microchannels were at the same scale as cells, single-cell analyses were rapidly developed. Furthermore, several studies focused on the miniaturization of molecular biology assays (polymerase chain reaction (PCR), cell lysis, DNA extraction and electrophoresis) following by their integration onto one single chip in which every step, from the sample collection to the final analysis, would be carried out in sequence. These devices gained popularity for their great potential for genomic analysis, being named Micro Total Analysis Systems ( $\mu \mathrm{TAS})$.

In parallel, microbioreactors finally emerged as a consequence of the application of microfluidics in the biological field. Because these devices are easily able to mimic chemical and biological environments, their main application concentrated into microanalysis concerning molecular biology (Whitesides, 2006). In the early 2000 s, some researches focused their efforts on the idea of using microbioreactors applied to industrial bioprocesses (Kostov et al. 2001; Maharbiz et al. 2004; Szita et al. 2005; Zanzotto et al. 2004; Zhang et al. 2006). In order to do so, microbioreactors were developed and evaluated by analysing parameters of industrial relevance such as growth rate, dissolved oxygen (DO), temperature and $\mathrm{pH}$ levels (Kostov et al., 2001; Maharbiz et al., 2004; Zanzotto et al., 2004; Szita et al., 2005; Zhang et al., 2006). Although the feasibility of applying such technology in the bioprocess field has been beautifully proven, the main limitations impeding further developments rely on instrumentation: the detection and control of parameters under the micro scale depends on micro tools, some of which are still in the process of development and may challenge the full capability of controlling very specific compounds.

Even though the scale down of industrial bioprocesses by using microbioreactors has proven to be an attractive and feasible option for experimental analyses, few research groups are familiarized with such technology. If microbioreactors are said to be promising for experimental analyses, with so many advantages over disadvantages, the question as to why they are still unpopular among bioprocess laboratories emerges. Whitesides posed this same question back in 2006 regarding microfluidics technology (Whitesides, 2006). Perhaps, according to Ho et al. (2015), such technology has yet to be explored for the development of a supreme application that could transcend the conventional approaches currently adopted.

With such concerns in mind, a literature search was carried out about microbioreactors in order to analyse the impact of this technology on the scientific scenario (Figure 1). Employing the Elsevier

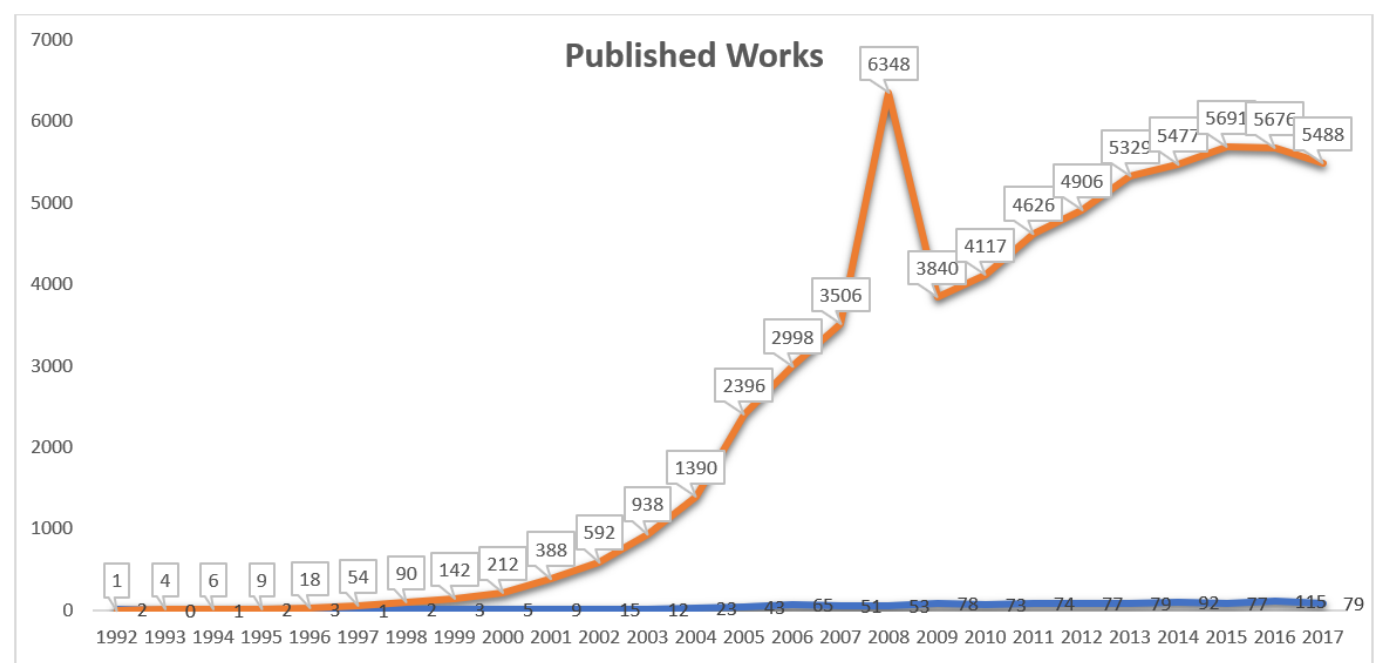

Figure 1. Correlation of the number of scientific publications over the years concerning microbioreactor technology (blue) and microfluidics technology (orange). This search was carried out using the analytical search tool from the Scopus platform (Elsevier B.V.) by the following strategies: "(microbioreactor*) OR (microfermentor*) OR (miniaturized AND bioreactor*) OR (microfluidic AND bioreactor*)" for article title, abstract and keywords; and "(microfluidic*)" for article title, abstract and keywords. 
database Scopus, when analysing the search strategy: "(microbioreactor*) OR (microfermentor*) OR (miniaturized AND bioreactor*) OR (microfluidic AND bioreactor*)" for article title, abstract and keywords, in comparison to "(microfluidic*)" for article title, abstract and keywords, there were only 1043 published works addressing microbioreactors out of nearly 65 thousand publications concerning microfluidics technology. This brief search evidences microbioreactors as a youthful technology with a growing influence over chemical and biochemical processes since the 1990 decade. Regarding the applications of microbioreactors within industrial bioprocesses and fermentations, such results thus indicate the great potential of an unexplored research field. Furthermore, Oliveira et al. (2016) carried out an analogous search concerning microfluidic approaches. Their study reported the availability of untouched possibilities for research not only for the application of microbioreactors in bioprocesses but also the general approaches of microfluidics in industrial biotechnology. Regardless of the rapid growth of this field of study, microfluidics has been a subject of concern from specific engineering journals, denouncing the complexity found when applying such technology to various other fields of study or handled by non-experts (Oliveira et al., 2016).

\section{MICROFLUIDICS}

A consequence of the advent of microtechnology, microfluidics composes a science that studies and manipulatesfluids insmallvolumes throughmicrometric channels (Breslauer et al., 2006; Whitesides, 2006; Ho et al., 2015). Microbioreactors have their origin as a derivation line from microfluidics, constituting one of the many approaches this technology might provide.

Because it is based on the reduction of the working volume, microfluidics consists of a sensitive approach, being susceptible to minor disturbances in the system. Such sensibility thus provides a high level of resolution and accuracy regarding the reliability and quality of obtained data. Therefore, microfluidics initially tended to analytical techniques as it could conduct experiments through a fast, cheap, accurate and sensitive approach, fulfilling the needs of experimental analyses (Whitesides, 2006). Because of the high resolution and sensibility, microfluidics may allow a better control of molecules within space and time (Whitesides, 2006; Szita et al., 2010). Importantly, a consequence of reducing the dimensional space is the increase of the surface-to-volume ratio and laminar flow, which reflects on the increase of heat and mass transfer phenomenon (Schäpper et al., 2009; Björnmalm et al., 2014; Sackmann et al., 2014). This might be positive or negative since reactions on the micro scale may occur faster and more accurate, but may also be affected by minor phenomena that usually are not vital problems at conventional scales (e.g. nonspecific adsorption by the prototype walls, evaporation and bubble formation).

Consequently, microfluidic devices present many features that are perfectly adapted to the needs of not only chemical and biochemical analyses, but also to a vast pool of applications in biology. The possibilities of controlling fluid dynamics and applying to microsystems can be very useful for the investigation of genetic responses to environment changes (Tay et al., 2010; Li et al., 2016b; Chiang et al., 2017), diagnoses (Chin et al., 2011; Huo et al., 2015; Patou et al., 2015; Li et al., 2016a), molecular screening (DeMello, 2006; Dittrich and Manz, 2006; $\mathrm{Yu}$ et al., 2014), microbial screening (El-Ali et al., 2006; Stott et al., 2010; Sjostrom et al., 2014; Jacques et al., 2017; Xie et al., 2017), simulation of cellular microenvironments (Chang et al., 2005; Sung and Shuler, 2009; Houshmand et al., 2016). Microfluidics is also a great strategy for the study of the omics sciences: genomics and transcriptomics (Wang and Bodovitz, 2010; Shalek et al., 2014; Macosko et al., 2015; Kim et al., 2016), proteomics (Sanders and Manz, 2000; Wang and Bodovitz, 2010; Wang and Yang, 2016) and metabolomics (Wang and Bodovitz, 2010; de Raad et al., 2016; Maisch et al., 2016), revolutionizing the methods for single cell studies and, therefore, the understanding of cellular properties, behaviour and cell heterogeneity.

In order to understand and manipulate the applications of microfluidic approaches in biology, a scientist must comprehend a range of multidisciplinary knowledge that addresses physics, chemistry and biology. Thereby, several concepts of fluid mechanics, geometry, heat and mass transfer, chemical interaction and biological behaviour constitute the pillars of the microfluidic approach in biological systems. The following topics will therefore discuss the major factors concerning microfluidic approaches and fabrications, such as the physical forces and phenomena, material composition, fabrication techniques, geometry configurations and their approaches.

\section{Physical Forces}

Within the microsystems, because of the small working volume applied, inertial and gravitational forces are less relevant, giving space to the regime of interfacial, convection and viscous forces (Fernandes et al., 2011; Marques and Fernandes, 2011). Furthermore, homogeneity is rapidly achieved by simple molecular diffusion considering the small size of microchannels and the consequential predominance of laminar flow 
(Schäpper et al., 2009). There are four principal dimensionless numbers that explore such physical forces: Reynolds number $(R e)$, capillary number $(C a)$, Weber number $(\mathrm{We})$ and Péclet number $(\mathrm{Pe})$.

Given the correlation between inertial and viscous forces, the Reynolds number $(R e)$ expresses the limitations of a laminar to turbulent flow:

$$
\operatorname{Re}=\frac{\rho \cdot v \cdot L}{\mu}
$$

where $\rho$ represents the fluid density $\left(\mathrm{kg} / \mathrm{m}^{3}\right), v$ the fluid velocity $(\mathrm{m} / \mathrm{s}), L$ the hydraulic diameter of the given microchannel (m) and $\mu$ the fluid viscosity (Pa.s). Microfluidic devices generally work with a $R e$ of approximately $10^{-1}$, in laminar flow (Beebe et al., 2002; Squires e Quake, 2005).

The capillary number $(\mathrm{Ca})$ characterizes the correlation between viscous and interfacial forces:

$$
C a=\frac{\mu \cdot V}{\gamma}
$$

whereupon $\mu$ represents the fluid viscosity (Pa.s), $V$ the flow velocity $(\mathrm{m} / \mathrm{s})$ and $\gamma$ the interfacial tension between two phases $(\mathrm{N} / \mathrm{m})$. In microfluidics, $\mathrm{Ca}$ is frequently lower than 1 , varying from 10 to $10^{-3}$, distinguishing patterns of biphasic flows. It is important to highlight the importance of this number to microdevices based on droplet generation because it characterises the capillarity behaviour, a phenomenon responsible for the generation of spherical drops (Berthier e Silberzan, 2009; Baroud et al., 2010). Droplet-based microdevices are discussed further later on in this review.

Based on both Re and $\mathrm{Ca}$, the Weber number $(\mathrm{We})$ correlates inertial forces to interfacial tension:

$W e=R e \cdot C a=\frac{\rho \cdot v^{2} \cdot L}{\gamma}$

where $\rho$ represents the fluid density $\left(\mathrm{kg} / \mathrm{m}^{3}\right), v$ the fluid velocity $(\mathrm{m} / \mathrm{s}), L$ the hydraulic diameter of the given microchannel $(\mathrm{m})$ and $\gamma$ the interfacial tension between two phases $(\mathrm{N} / \mathrm{m})$. We is an interesting parameter because inertial forces are less relevant in microfluidic devices, maintaining the configuration of the fluid interface and thus forming droplets at regular intervals (Berthier and Silberzan, 2009; Basova and Foret, 2014). Therefore, $W e$ is another dimensionless number of substantial importance for droplet-based microdevices.

Finally, the Péclet number $(\mathrm{Pe})$ correlates the convection force to the diffusion phenomenon. It is used to characterise the mass transport within systems:
$P e=\frac{v \cdot L}{D}$

where $v$ represents the fluid velocity $(\mathrm{m} / \mathrm{s}), L$ the hydraulic diameter of the given microchannel $(\mathrm{m})$ and $D$ the diffusive coefficient. In microfluidic devices, the flow velocity is usually small, resulting in small $P e$ numbers, which indicates the dominance of a diffusion process over mixing by convection (Livak-Dahl et al., 2011; Marques and Fernandes, 2011).

Other dimensionless numbers such as Bond $(B o)$, Ohnesorge $(O h)$ and Damköler $(D a)$ are also applied in microfluidic studies regarding the correlations of gravitational to surface forces, viscous to inertial forces and transport to reaction timing, respectively (Marques and Fernandes, 2011).

\section{Material Composition and Microfabrication Methods}

Microfluidic devices are versatile largely customized platforms and may vary in material composition (Table 1) and geometry configuration (posteriorly discussed), depending on the peculiarities and exigencies of the process or technique involved. Each material has its own properties, requiring different strategies for the microfabrication process. In the light of such matters, it is of utter importance to consider what is the purpose of a respective device when considering a particular material for its fabrication, because different attributes may limit expected outcomes. Examples of important attributes to be considered are translucency, gas permeability, flexibility, chemical endurance, biocompatibility, solvent compatibility and nonspecific adsorptions (McDonald and Whitesides, 2002; Schäpper et al., 2009; Qin et al., 2010; Wolfe et al., 2010). Even more, the level of integration and instrumentation on a device is proportional to the complexity and specificity of the microfabrication process, establishing a tradeoff between the capacity of sensing and controlling variables and microfabrication feasibility (Schäpper et al., 2009).

The very first materials adopted for the construction of microfluidic prototypes were silicon and glass, two outstanding options for microreactions and analyses because they present great thermoconductivity, rapid capillary electrophoresis, solvent tolerance and adequate strength (McDonald and Whitesides, 2002; Nge et al., 2013). Nevertheless, these materials were rapidly replaced by a variety of other options. The reason relies mainly on the inflexibilities found during the conductivity of the microfabrication process using such materials, consisting of a time-consuming and expensive process, also requiring clean room facilities (Schäpper et al., 2009; Nge et al., 2013). Furthermore, 
Table 1. Materials used for microfabrication and their principal characteristics.

\begin{tabular}{|c|c|c|}
\hline Material & Characteristics and Applications & Reference \\
\hline Ceramic & $\begin{array}{l}\text { Presents good chemical and thermal resistance. On the other hand, as an } \\
\text { opaque material, low visibility is available, limiting observation and optical } \\
\text { detection. }\end{array}$ & $\begin{array}{c}\text { Belavic et al. (2016) } \\
\text { Czok and Golonka (2016) } \\
\text { Groß et al. (2008) } \\
\text { Peterson et al. (2005) } \\
\text { Vasudev et al. (2013) }\end{array}$ \\
\hline Glass & $\begin{array}{l}\text { Has a good overall chemical resistance allied with great capacity for } \\
\text { visualisation. However, it is difficult to work with and may not be applie d to } \\
\text { strong aqueous bases due to its incompatibility. Microfabrication might be } \\
\text { less flexible, expensive and requires clean-room facilities. }\end{array}$ & $\begin{array}{l}\text { Zeibi Shirejini and Mohammadi (2017) } \\
\text { Vladisavljević et al. (2014) } \\
\text { Nabavi et al. (2015) } \\
\text { Othman et al. (2015) } \\
\text { Petrucci et al. (2017) }\end{array}$ \\
\hline Paper & $\begin{array}{l}\text { Simple fabrication process, inexpensive, do not require additional } \\
\text { instrumentation, ubiquitous and easy to handle. Nevertheless, the application } \\
\text { of such devices is strongly limited. }\end{array}$ & $\begin{array}{l}\text { Martinez et al. (2008) } \\
\text { Martinez et al. (2010) } \\
\text { Jiang and Fan (2016) } \\
\text { Cao et al. (2017) } \\
\text { Lam et al. (2017) } \\
\end{array}$ \\
\hline PDMS & $\begin{array}{l}\text { Inexpensive and very flexible for applications. It also has good optical clarity. } \\
\text { Easy to work with and has reduced time of manufacturing. It is gas permeable, } \\
\text { non-toxic and has a low elastic modulus enabling integration. }\end{array}$ & $\begin{array}{l}\text { McDonald and Whitesides (2002) } \\
\text { Sia and Whitesides (2003) } \\
\text { Chiang et al. (2017) } \\
\text { Dong et al. (2017) } \\
\text { Vittayarukskul and Lee (2017) } \\
\end{array}$ \\
\hline PMMA & $\begin{array}{l}\text { Presents biological compatibility, good visibility for detection and ease of } \\
\text { microfabricating. It is also impermeable to gases and does not support high- } \\
\text { level temperatures. }\end{array}$ & $\begin{array}{l}\text { Adams et al. (2008) } \\
\text { Asl et al. (2016) } \\
\text { Li et al. (2016) } \\
\text { Van Anh et al. (2016) } \\
\end{array}$ \\
\hline Silicon & $\begin{array}{l}\text { It supports high levels of temperature and pressure and has a great } \\
\text { thermoconductivity. Microfabrication might be time consuming and } \\
\text { expensive. Not compatible with strong aqueous bases and detection via light- } \\
\text { emitting diodes (LEDs) might be challenging due to the low light visibility. }\end{array}$ & $\begin{array}{l}\text { Cady et al. (2003) } \\
\text { Chen et al. (2006) } \\
\text { Chandrasekaran et al. (2007) }\end{array}$ \\
\hline $\begin{array}{l}\text { Stainless } \\
\text { steel }\end{array}$ & $\begin{array}{l}\text { A good option for thermal conductivity, supporting high levels of temperature } \\
\text { and pressure. On the other hand, it is sensitive to corrosion and, as an opaque } \\
\text { material, there is no visibility for detection. }\end{array}$ & $\begin{array}{l}\text { Tong et al. (2001) } \\
\text { Kobayashi et al. (2008) }\end{array}$ \\
\hline Teflon & $\begin{array}{l}\text { Considerably less utilized, it presents a great tolerance over solvents and } \\
\text { general chemicals, high biocompatibility and is gas permeable. }\end{array}$ & $\begin{array}{l}\text { de Haas et al. (2012) } \\
\text { Zheng et al. (2013) } \\
\text { Walsh et al. (2016) } \\
\text { Ren et al. (2011) }\end{array}$ \\
\hline
\end{tabular}

silicon and glass are not permeable to gases and hence may not be applicable to prolonged reactions.

As the technology developed, polymers such as poly(dimethylsiloxane) (PDMS) and poly(methyl 2-methylpropenoate) (PMMA) became progressively popular as less onerous substrates for microfabrication (Nge et al., 2013). The elastomer PDMS is an inexpensive and nontoxic versatile option, enabling a rapid construction of microdevices via soft lithography (McDonald and Whitesides, 2002). It also has a low Modulus of Elasticity, an important feature for the versatility of this material, allowing the integration of microfluidic devices such as pneumatic pumps, valves and mixers within the scope of the prototype (Qin et al., 2010; Nge et al., 2013). Even though the integration level is proportional to the complexity of the design and fabrication of a prototype, as discussed before, integration has a significant importance on the efficiency and on the expenses of the device since better control of parameters is reached and fewer macroscale devices are required for this task (Schäpper et al., 2009). Nevertheless, the polymer PDMS may be incompatible with some solvents as it swells in the presence of such components. It is also permeable to gases, an important feature for bioreactions involving transport and gas exchange, which can be beneficial (e.g., in gas exchanges and prolonged cell cultures) or detrimental (e.g., in evaporation, an important issue for microreactions regarding the size of the working volume) (Schäpper et al., 2009). The elastomer PMMA on the other hand, has impermeability to gases, constituting an easy alternative for micromachining (under low levels of temperature, e.g., around $100^{\circ} \mathrm{C}$ ) (Nge et al., 2013). Importantly, both PDMS and PMMA are transparent, favouring optical observation and detection via light emission apparatus. Further information about the various materials and their properties applied to microfabrication and microfluidics is widely described by Nge, Rogers, and Woolley (2013).

When fabricating a microfluidic device, the technique applied might depend on the material used as substrate (composition of the future device). In general, photolithography is the most popular methodology for prototyping, constituting a fast and precise technique that has a great history in the development 
of microtechnology. Nevertheless, this method is inevitably expensive since it demands particular equipment, clean room facilities and the development of specific photomasks, limiting the application to only photosensitive polymers (Qin et al., 2010; Wolfe et al., 2010). It is also time-consuming, does not grant control over interfacial chemistry and may only be applied to planar substrates (Qin et al., 2010). Alternatively, soft lithography, an adaption of the classic lithography for soft substrates (e.g. polymers), is an interesting approach for a rapid prototyping method with a large number of patterning techniques (Addae-Mensah et al., 2010). Unlike photolithography, soft lithography is a versatile approach, allowing the application of soft, flexible, curved and planar substrates, addressing low expenses and precision on both micro- and nanoscales (Xia and Whitesides, 1998; Qin et al., 2010).

According to Weibel, Diluzio and Whitesides (2007), a rapid and popular example of the step-by-step process of soft lithography for microfabrication using PDMS as substrate is schematized in Figure 2 (Weibel et al., 2007). At a first instance, the design and function of the device might be idealized corresponding to the needs and specifications of the process involved. After

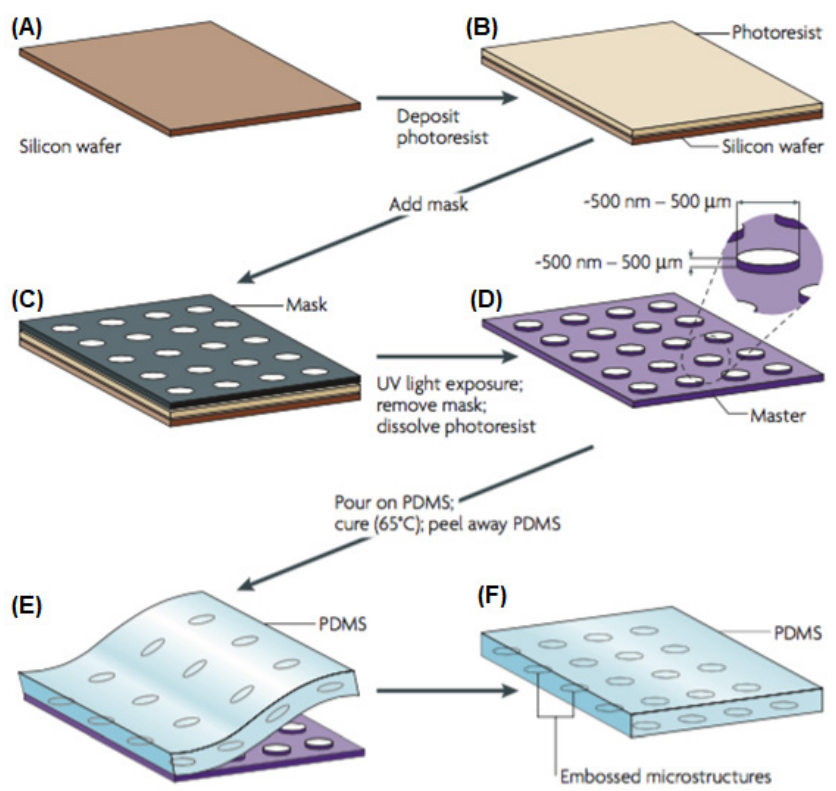

Figure 2. Example of a patterning protocol using soft lithography for the fabrication of micropatterned slabs using PDMS as substrate. Uniformization of photoresist by coating on a silicon wafer (A and $\mathrm{B})$. The mask is placed over the photoresist $(\mathrm{C})$. The system is exposed to UV light and non-polymerized photoresist is washed out by a solvent, rising the master piece (D). The substrate (PDMS) is applied over the master and cured thermally (E). The final piece is peeled away with the microstructures embossed into its surface (F). Reprinted by permission from Macmillan Publishers Ltd: Nature Reviews Microbiology (Weibel et al., 2007), copyright 2007. the idealization, technical drawings are created with the help of computational tools, such as computer-aided design (CAD) software. The drawings are then used for the construction of a mask, which is responsible for the modulation of channels. Deposited on a silicon wafer, a photosensitive polymer, called photoresist, is spin-coated and then covered by the mask. In sequence, the photoresist is exposed to ultraviolet (UV) light through the mask, allowing the polymerization of the exposed polymer. To remove the photoresist that has not reacted with the UV light (crosslinked), an organic solvent is applied, leaving the piece of photoresist patterned according to the mask, also called master piece. The height of the device is controlled by the amount of substrate (thickness) applied over the wafer and the mask transfers the shape and lateral information to the master piece. Therefore, the master may contain all the detailed dimensional features first idealized. The substrate (PDMS in this case) is thus applied in the master and cured thermally, resulting thereafter in a PDMS layer with the respective microstructure embossed in the surface.

In terms of the industrial baseline, thermoplastics such as PDMS and PMMA may be cost-effective but with the absence of a fast and robust process of fabrication. Even though soft lithography is a trend technique for laboratorial prototyping using PDMS, it still requires the master structure, which can demand an inconvenient amount of time to fabricate on the industrial level (Kotz et al., 2017). An alternative solution consists of adopting direct structuring approaches that use photocurable polymers able to be structured by light without the need of a master piece. However, the number of adequate materials for these techniques is still limited, comprehending, most of the time, resins of undefined composition. As a consequence, it is extremely difficult to conduct the direct transference of prototypes on the laboratorial scale to the industrial state (Kotz et al., 2017).

Recently, the advent and popularization of 3D printing techniques have been attracting the attention of many researchers by their capability of developing complex structures with accurate resolution (Tseng et al., 2014; Ho et al., 2015; Au et al., 2016; He et al., 2016). Popular techniques such as stereolithography and laminated object manufacturing (LOM) may allow a regular fabrication of multiple prototypes, demanding less time and less manual handling during the process (Ho et al., 2015). Therefore, those with less expertise in microfabrication processes may be capable of accurately fabricating microfluidic devices in short periods of time. Further information regarding 3D printing and the different methods applied for the fabrication of microfluidic devices may be accessed in Ho et al. (2015).

Finally, the last step of the microfabrication procedure consists of the sealing process. This step is 
responsible for assembling all the parts and components of the microdevice into one final piece: the device itself; guaranteeing no leakage of inner fluids during reactions. The sealing process might be reversible or irreversible and this will depend on the material that composes the microdevice, as well as the material and method used for sealing. Therefore, some materials might imply more or less laborious sealing processes, directly influencing the feasibility and practicality of the device.

\section{Geometric Configurations and Approaches}

The application of microfluidic systems in chemical biology has increased throughout the years, reaching a significant level of approaches and applications. Indeed, the possibilities are enormous regarding the variety of geometric forms and integration options. A scientist willing to succeed in the world of microfluidics might have to deal not only with an extended level of knowledge about physics, chemistry and microfluidics itself, but also a great parcel of creativity regarding possibilities and applications. Like microbioreactors, several other microfluidic approaches have been gradually making space in the world of science. In consideration of such fact, some of the popular approaches based on microfluidic technology are hereby presented.

Microarrays - Probably the most intuitive approach of microfluidics, microdevices that are comprised of whole sets of intercepted microchannels are useful when aiming to investigate the behaviour and interactions between cells, proteins and other molecules (Weibel and Whitesides, 2006). Therefore, applications are intensively variable, transiting from general molecular biology assays to point-of-care diagnosis. Gao et al. (2017), for example, developed a real-time assay capable of detecting lung cancer biomarkers. The device was constructed based on PDMS chips and contains two divided chambers for rapid analytical responses. A second example is the advance in genome mapping techniques described by Lam et al. (2012). In this work, fluorescent labelled sequence motifs were used for the construction of maps able to indicate physical distances between sequences. In a range translating from the early to the latest published works, some other applications may comprehend the combination of fluids for biochemical reaction studies (Ismagilov et al., 2001), pattering the delivery of chemical reactants (Delamarche et al., 1998), isolation of single-cell mRNAs (Marcus et al., 2006) and on-chip power generation based on microbial fuel cells (Yang et al., 2017) (Figure 3). As can be seen, microdevices based on microarrays may be carefully planned regarding the purpose and application involved. Therefore, each configuration emerges in the aftermath of the application requirements.

Concentration Gradient - Microfluidics allows the manipulation and control of fluid streams with laminar behaviour, enabling the prototyping of microdevices capable of generating concentration gradients of specific molecules like no other technique (Weibel and Whitesides, 2006) (Figure 4). Therefore, gradient generators can often simulate complex biological microenvironments, allowing the study of cellular responses to the most various conditions in vivo. There are two major groups of such devices: diffusion-based gradient generators (Keenan et al., 2006; Mosadegh et al., 2007; Atencia et al., 2012) and flow-based gradient generators (Jeon et al., 2000; Cooksey et al., 2009; Xu et al., 2016). The principal difference between these groups relies on the physical principle of gradient formation: as diffusion-based gradients

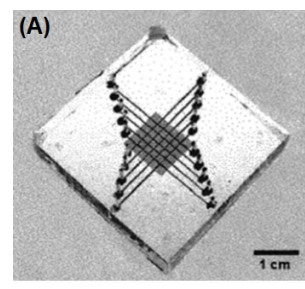

(B)

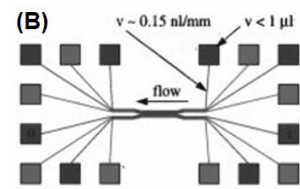

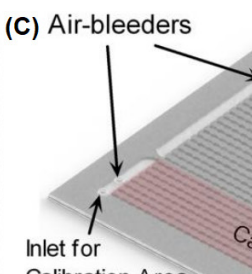

Inlet for
Calibration Area

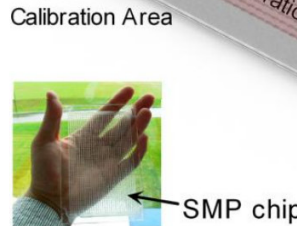$$
\text { SMP chip }
$$

Main Inlet

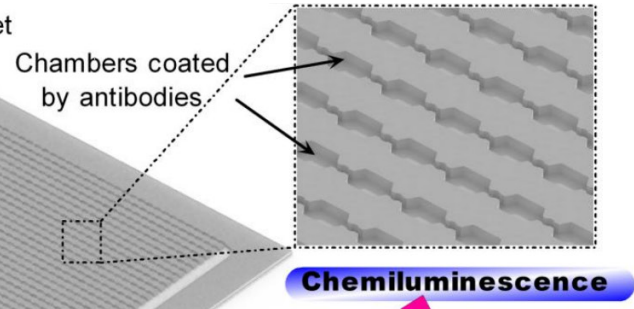

Chambers coated

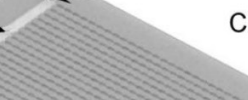

Figure 3. Microfluidic devices based on microarrays: (A) microfluidic system with parallel arrays for the fluidfluid diffusional contact study for biochemical reactions. [Reproduced from Ismagilov et al. (2001) with permission of the American Chemical Society]. (B) schematic design of a microfluidic network of channels for the concomitant transportation of reactants from peripheric macroscopic pads to a central microchannel where detectors are located. [Reproduced with permission from Delamarche et al. (1998) with permission of the American Chemical Society] (C) microfluidic system with 1536 chambers for mood-disorders-related-serological studies based on chemi-luminescent immunoassays as illustrated in the bottom right corner. A photo of the system is provided at the bottom left corner. [Reproduced from Zhao and Dong (2013), published under Creative Commons Attribution License (CC BY 3.0)]. 
(A)

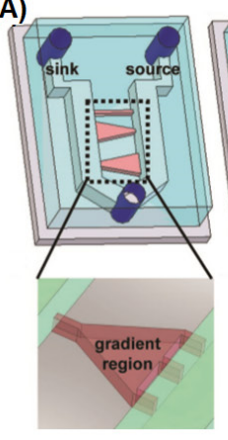

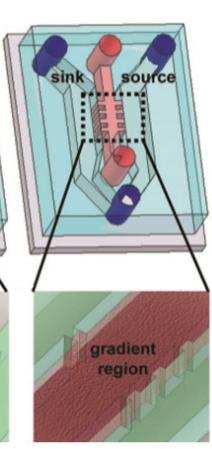

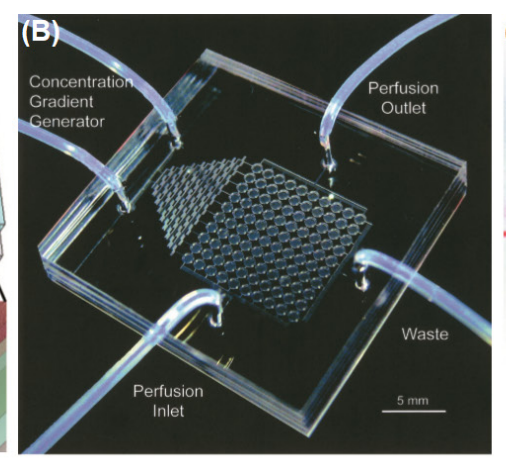

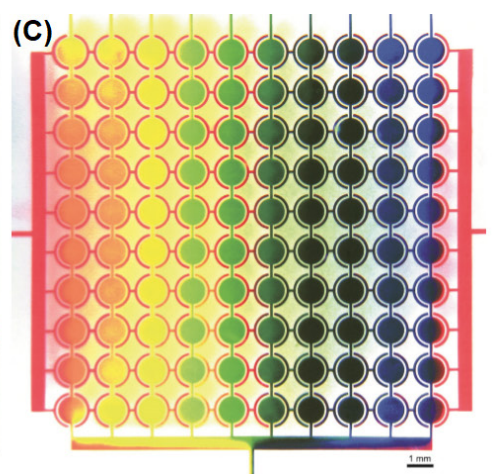

Figure 4. Concentration gradient microfluidic devices: (A) scheme of a concentration gradient-based microdevice composed of two inlets, a sink channel (lower or no concentration) and a source channel (higher or total concentration), along with red regions where nonlinear gradients are formed due to the asymmetric configurations. [Reproduced with permission from Mosadegh et al. (2007). Copyright 2007 American Chemical Society]. (B) microfluidic cell culture device combining both array and concentration gradient approaches, composed of inlets and outlet of media (left and right ports) and inlet and outlet of cells and reagents (top and bottom ports). (C) colorimetric record of the concentration gradient formed by the device. [Reproduced with permission from Hung et al. (2005). Copyright 2005 Wiley Periodicals].

are formed by passive diffusion with no fluid flow and generate a linear gradient, flow-based gradients are formed by the diffusion of two or more laminar stream flows at their interface, generating a non-linear gradient (Toh et al., 2014). Both approaches are able to investigate biological responses, but the continuous flow present in flow-based gradient generators might disturb cell communication as molecular factors might be washed out (Saka et al., 2017). Gradientgenerator microdevices have been employed in a number of biological applications, including studies on chemotaxis (Wolfram et al., 2016), investigations on pharmacological activity (Pihl et al., 2005), stem cell differentiation (Chung et al., 2005), cell culture arrays (Hung et al., 2005) and systems-biology of microorganisms (Breslauer et al., 2006; Lee et al., 2006). Further information about microfluidic gradient generators concerning biological applications can be accessed in Kim et al. (2010) and Toh et al. (2014).

Not restricted to molecular gradients, thermal gradients (Lucchetta et al., 2005; Baranek et al., 2010) can also be generated using microdevices. Baranek et al. (2010) developed a microfluidic device for the temperature stimulation of Caenorhabditis elegans embryonic cells based on a thermal gradient. This prototype led to the manipulation of cell division rate within in vitro cultures. Analogously, Lucchetta et al. (2005) developed a similar device for the evaluation of thermal effects on embryonic development of Drosophila melanogaster.

The application of concentration gradient devices is very interesting from the point of view of fermentative bioprocesses as they could become a practical tool for quick investigation of cellular behaviour under the regime of several variables such as substrate and solvent concentration, temperature and $\mathrm{pH}$ levels.
Single-cell Trapping Microarrays - Microdevices based on single-cell microarrays are microfluidic tools able to isolate and trap single cells (Figure 5), which have been arousing the interest of many researchers over the past years because of its attributes. The study of cellular behaviour in conventional culture dishes or wells is not as precise as using single-cell microarrays because accuracy is inversely proportional to cell-tovolume ratio. For example, inhomogeneity may cause
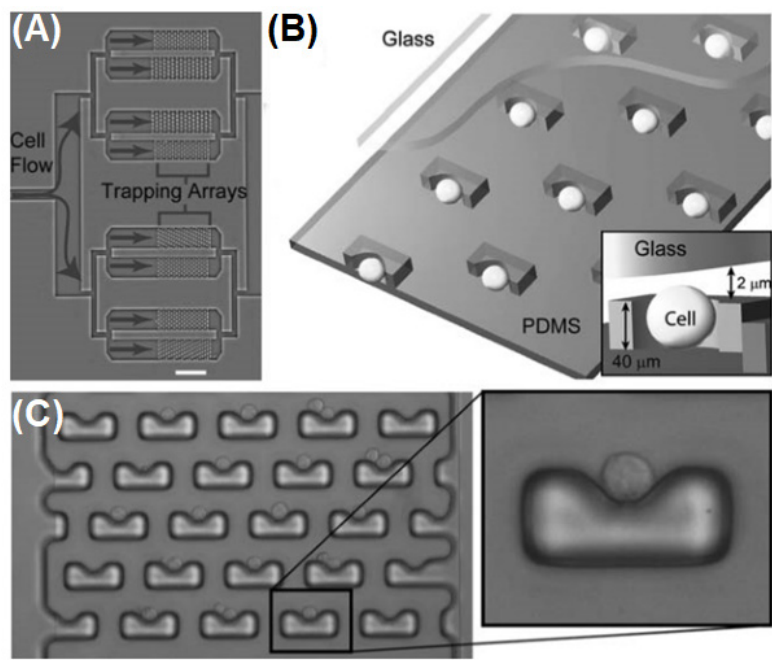

Figure 5. Single-cell trapping-based approaches: single cell trapping array: (A) photograph of the device, indicating the flow direction containing cell and media to the distribution through the trapping chambers. (B) schematic 3D diagram of the device illustrating the principle of the mechanism of cell entrapment. (C) a bright-field micrograph of the trapping chambers showing entrapped cells, where in some cases two cells are found to be entrapped together. [Reproduced from Di Carlo, Wu, and Lee (2006) with permission of The Royal Society of Chemistry]. 
the misinterpretation of cell responses regarding the concentration of cells in particular spots within a culture, reducing nutrient availability and increasing waste content (Di Carlo et al., 2006). Regarding such aspects, cell-trapping microarrays may overcome inhomogeneity, as cells are isolated from one another. Therefore, this approach allows various cell biology investigations such as biological responses of cells to environmental stimulus (Eriksson et al., 2007), drug screening (Wlodkowic et al., 2009), gene expression (Toriello et al., 2008), metabolism (Maglica et al., 2015) and pharmacokinetics (Luo et al., 2012).

Grünberger, Wiechert, and Kohlheyer (2014) recently reviewed the applications of single-cell microdevices in the development of bioprocesses. Such prototypes may offer knowledge on microorganism growth and morphology, strain and process characterization, population heterogeneity and environmental dynamics. Consequently, microfluidic single-cell devices may perform as analytical tools with potential to increase the understanding of microorganism performance under industrial conditions with an ultimate application in bioprocess optimizations.

Droplet Microfluidics - Droplet-based microfluidics is the strategy of generating discrete droplets within distinguished patterned biphasic flows (Figure 6). Usually, a hydrophobic fluid is adopted as a continuous phase and an aqueous solution is used to

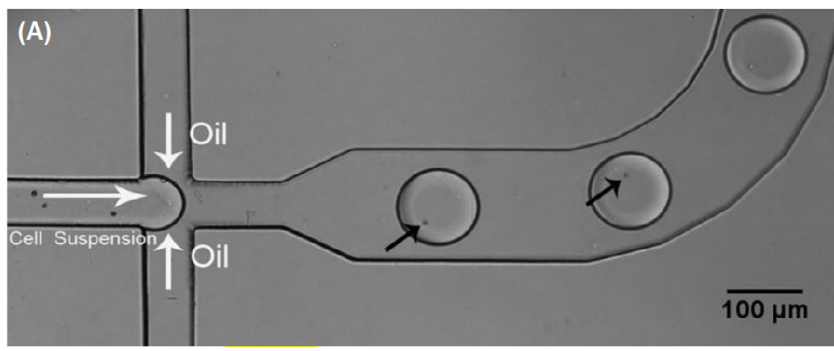

(B)

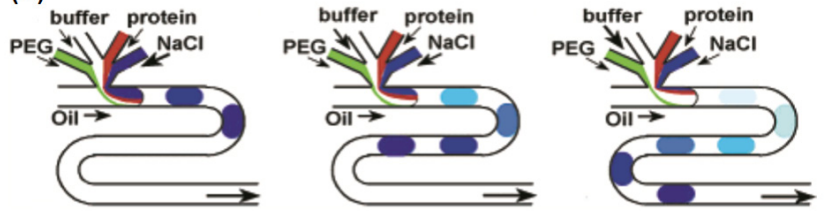

Figure 6. Droplet microfluidic devices: (A). brightfield micrography of algal cells (black arrows) encapsulated in droplets. The cell suspension and oil flow are indicated by white arrows. [Reproduced from Pan et al. (2011) with permission of The Royal Society of Chemistry]. (B) illustration of the formation of droplets with different concentration of $\mathrm{NaCl}$ as its flow rate decreases, resulting in a different microenvironment in each droplet applied to the crystallization of proteins. [Reproduced with permission from Zheng, Roach, and Ismagilov (2003), Copyright 2003 American Chemical Society]. compose the droplets (Teh et al., 2008). The continuous phase, initially separated in a first microchannel, has a high flow velocity while the aqueous phase, in another microchannel, has a much lower flow velocity. When both microchannels are integrated, the aqueous phase is split into small drops, characterising the phenomenon of droplet formation. When designing a strategy for the generation of droplet-based microdevices, a researcher might give special attention to the material composition of the device, geometry and fluid composition (Teh et al., 2008; Baroud et al., 2010). Such aspects may influence the droplet formation, stability of fluid interface, size of droplets or even provoke coalescence (Teh et al., 2008; Baroud et al., 2010; Baret, 2012). Therefore, the dimensionless numbers $\mathrm{Ca}$ and We are largely applied in this approach regarding the capillarity behaviour within fluids of different compositions and therefore different physical properties (Berthier e Silberzan, 2009).

In such an approach, the formation of droplets can isolate reagent material within them, turning each droplet into a tiny singular reactor. Reagents can vary from cells to chemicals and therefore several applications emerge for chemical and biological approaches (Fair et al., 2007; Huebner et al., 2008), including screening of conditions for crystallization (Zheng et al., 2003), point of care testing (Sista et al., 2008) and systems and synthetic biology development (Szita et al., 2010). Furthermore, by parallelizing multiple layers of droplet generators, Conchouso et al. (2014) achieved production yields applicable to the industrial scale. Nevertheless, such devices still require extensive revision for their consolidation as an efficient industrial tool (Holtze, 2013).

\section{MICROBIOREACTORS APPLIED TO BIOPROCESSES}

The development of industrial bioprocesses is of great importance for the emergence of bioproducts with radical or incremental innovative applications, which could mean the solution for current issues in our society. Even though the behaviour of cells under industrial conditions has been widely studied over the past decades, the nature of biological systems is complex and might demand experimentation for comprehension under the various conditions applied in a bioprocess (Schmidell et al., 2001; Micheletti e Lye, 2006). When considering both upstream and downstream phases, the complexity level is expected to increase, demanding further efforts and studies. Moreover, the optimization of bioprocesses plays an important role in the capacity and feasibility of manufacturing such products, which also demands intensive screening and experimental tests. 
Resorting to the conventional bench scale for the study of bioprocesses is expensive and time-consuming, forcing many researchers to find shelter in statistical analyses that are not always supported by a proper number of experimental assays. Microbioreactors thus consist of a feasible solution, enabling the fast screening of a wide range of variables for the development or optimization of industrial bioprocesses. Nevertheless, it is important to understand that many parameters are not always directly translated to macroscales. Most of the advantages of microscale analysis, such as the fast transfer of heat and mass, may turn out to be limitations for the scaling-up step. Therefore, microbioreactors may be applied as preliminary tools in the study of bioprocesses with industrial perspectives.

In such aspects, researchers have not always used the concept of microbioreactors in concordance. Aiming to present and clarify the differences and peculiarities of microbioreactors, this part of the review will discuss the different concepts of microbioreactors (microwell and microfluidic bioreactors) and further particularities when transfering bioprocesses to the microscale, including heat and mass transfer and detection and control of parameters.

\section{Microwell Bioreactors and Microfluidic Bioreactors}

Microbioreactors can be divided into two distinct approaches: microwell bioreactors and microfluidic bioreactors (Micheletti and Lye, 2006). The first approach consists of adopting microliter plates, typically containing 24 or 96 wells with total working volume varying from microliters to millilitres, as platforms for bioreactions where each well mimics a single bioreactor. In this case, microliter plates are coupled to a system able to detect and control parameters. Such a system is also responsible for translating detection signals into computational data used for analyses. Blesken et al. (2016) recently reported a 48-well microliter plate platform (800 to $1500 \mu \mathrm{L}$ total filling volume) with an integrated microfluidic chip able to provide online data from the measurement of biomass, fluorescence, $\mathrm{pH}$ and dissolved oxygen (DO) levels with non-invasive methods. The system also enables automated feeding and $\mathrm{pH}$ control of fermentations. This is possible by using two well rows as reservoirs of feeding media and $\mathrm{pH}$ control solutions. Even though $\mathrm{pH}$ regulation using liquids is a practical approach, the fermentation solution might suffer a difference in the total volume, which would require caution and special attention during the fermentation and data analysis regarding the small working volume applied in microscale bioprocesses. Another relevant microplate system is the micro-Matrix (Applikon, Delft, The Netherlands), which is commercially available, offering a 24 well plate platform with independent measurements and control over $\mathrm{pH}$, temperature, $\mathrm{DO}$ and feeding mechanism.

Microplate systems can also be equipped with individual impellers for enhancement of the oxygen transfer coefficient $\left(K_{L} a\right)$ values (Micheletti e Lye, 2006). Puskeiler, Kaufmann, and Weuster-Botz (2005) developed a 48-well microliter plate with incorporated impellers, either free floating or supported by a hollow shaft, reaching $K_{t} a$ values of around $>0.4$ $\mathrm{s}^{-1}$. Commercially available microplate systems with automatic stirrers are also available. A relevant example is the bioREACTOR 48 from 2mag (Munich, Germany) with 48 wells with a working volume capacity from 8 to $15 \mathrm{~mL}$, automated with temperature control and non-invasive $\mathrm{pH}$ and DO measurements. This system is also capable of performing $K_{L} a$ values of $>0.4 \mathrm{~s}^{-1}$.

On the other hand, microfluidic bioreactors are engineered devices usually mimicking industrial scale bioreactors. Such devices are generally composed of a fermentation chamber highly integrated with sensors and controllers providing high-throughput analyses. Microfluidic bioreactors are thus a versatile approach commonly adopted during the screening of variables and conditions for the engineering and optimization of bioprocesses. For example, Zanzotto et al. (2004) described a microbioreactor with integrated aeration and on-line measurement of optical density (OD), DO and $\mathrm{pH}$ based on optical, non-invasive methods. The device was tested with bacterial fermentation and the results were positively compared with a bench-scale version of the same fermentation. Even though the majority of microfluidic bioreactors are designed for a specific type of bioreaction, $\mathrm{Au}$, Shih, and Wheeler (2011) developed a device capable of automated cultivation and density analysis of bacteria, algae and yeasts, calling it a BAY microbioreactor. This device was able to attend the singularities of each one of these microorganisms simultaneously by culturing cells in distinct droplets without using valves, pumps or mixers in compatibility with bench-top analysers $(\mathrm{Au}$, Shih, and Wheeler 2011).

Recently, Johnson-Chavarria et al. (2014) developed an automated single-cell microbioreactor capable of establishing a chemostatic environment, continuously delivering fresh nutrients and removing produced metabolites. Such a device is useful for the study of cellular behaviour over media exchanges and for continuous fermentation process analyses. The microbioreactor was integrated with computercontrolled pressure regulators and computer-controlled syringe pumps for pressure and media delivery control, along with a thermal plate heater for temperature regulation (Figure 7, panel A). The experimentation included the observation of cellular dynamics under 


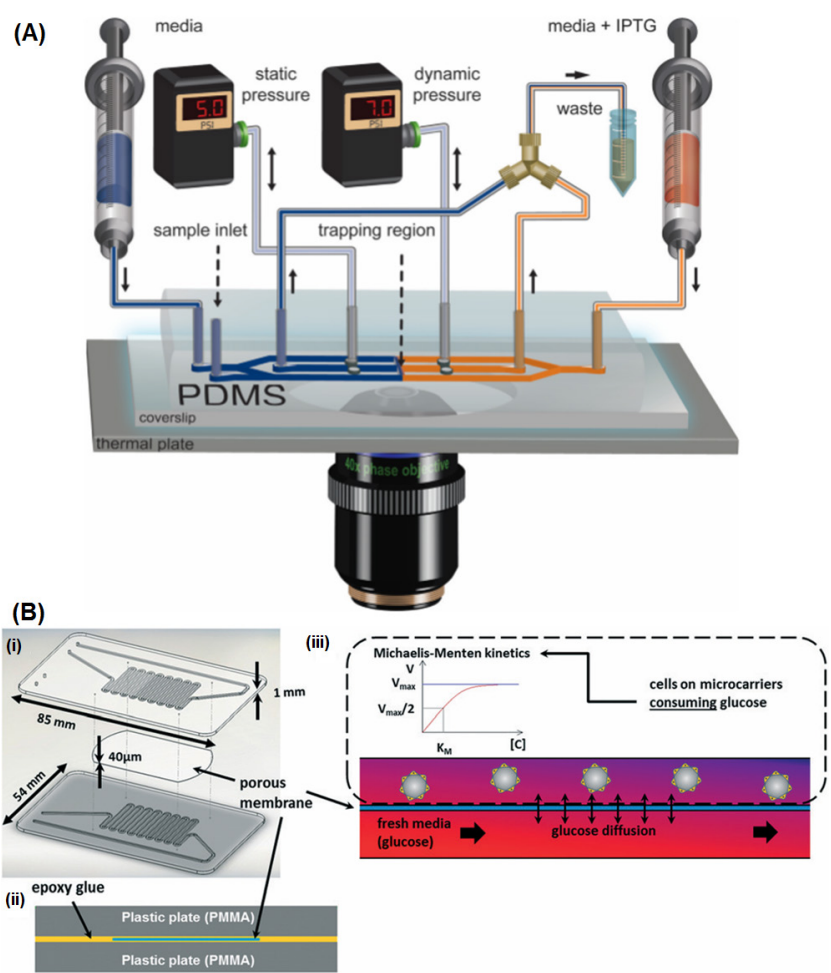

Figure 7. Microbioreactors as tools for continuous culture assays: (A) microbioreactor for continuous culture of single-cells, integrated with computercontrolled pressure regulators and syringe pumps for valve pressure and media delivery, respectively; a thermal plate equipped with thermocouple for temperature control and an inverted microscope equipped with fluorescent and phase-contrast imaging. The device is also equipped with a sample inlet. [Reproduced from Johnson-Chavarria et al. (2014) with permission of The Royal Society of Chemistry]. (B) schematic illustration of a microbioreactor for continuous culture of mammalian cells in microcarriers. (i) detailed dimensions of each piece that assemble the microbioreactor. (ii) illustration highlighting the porous membrane between the two PMMA pieces. (iii) scheme illustrating the principle of the microbioreactor, where the upper channel hosts mammalian cells consuming the substrate while the lower channel provides fresh medium by diffusion though the porous membrane. [Reproduced from Abeille et al. (2014) with permission of The Royal Society of Chemistry].

media exchange by Escherichia coli cells, using an inverted microscope equipped for phase-contrast and fluorescence imaging. Further developments on such a device might concern parallelization aiming to overcome low throughput analysis and a more sophisticated feedback controller concerning the confinement of multiple cells. A higher level of integration would also be interesting regarding other variables such as $\mathrm{pH}$ and dissolved oxygen concentrations.
In the same way, Abeille et al. (2014) developed a microbioreactor based on the continuous culture of mammalian cells in microcarriers. The device was made of PDMS and composed of two channels at different levels, assembled on a snake-like shape (Figure 7, panel B). A porous membrane is integrated between each channel, confining the cells in the first channel. Fresh media is supplied by a syringe-pump system, flowing through the second channel and diffusing through the pores of the membrane. Therefore, long-term bioprocesses may occur in such device, thus enhancing future developments in continuous mammalian cell cultures. The device was also integrated with a thermal sensor and temperature control could be achieved by placing the device in a heater apparatus. As for the experimentation, Drosophila S2 cells were monitored by a laser scanning confocal microscope. Some of the limitations of this device may consist of the controllability of the system as temperature was the only variable sensed, and cell harvesting protocols may be further enhanced regarding the nature of the established device (Abeille et al., 2014).

Different from the current trends in the microbioreactor world, Krull and Peterat (2016) accomplished an interesting work focusing not on the development process of microbioreactors, but on the feasibility and kinetic profiles of reactions of a multiphase microbioreactor for aerobic submerged continuous fermentation. Cells of Saccharomyces cerevisiae were analysed for chemostat cultivation and kinetic models were determined using experimental data from the stationary concentration of substrate, cells and ethanol. Linear methods such as Lineweaver-Burk, Eadie-Hofstee and Hanes Woolf were applied for the determination of the maximal specific growth rate $\left(\mu_{m a x}\right)$ and the Monod constant $K_{S}$. Yield coefficients $Y_{X / S}, Y_{P / S}$ and $Y_{P / X}$ were determined based on the model of Luedeking-Piret. Finally, the metabolism kinetic model was built based on two validity ranges considering the Crabtree effect: (i) $\mu=$ $D<D_{\text {crab }}$, where the metabolism is considered purely oxidative, converting substrate into biomass or applied for endogenous maintenance; and (ii) $\mu=D>D_{\text {craa }}$, where oxido-reductive metabolism is considered under the Crabtree effect, taking into account the conversion of substrate into ethanol and correlating it with the metabolic activity of the cells. The results obtained were compared to bench scale data for a volume around 50,000 times higher than that applied on the micro scale, in which both kinetic constants were of the same order, proving the feasibility and applicability of the microdevice as a tool for bioprocess studies.

\section{Microbioreactor Particularities}

Variables affecting bioprocesses usually consist of strain, media composition and physicochemical 
conditions such as DO level, temperature and $\mathrm{pH}$ level. Therefore, microbioreactors must be integrated or at least coupled to a platform able to detect and measure such parameters. Nevertheless, even the most integrated microbioreactor may face complications while carrying out fermentation processes regarding the reactor design, material composition and the more variable characteristics of the bioprocess itself (e.g., the operation modulus and aeration requirement). Furthermore, because of the small working volume, aspects such as heat and mass transfer, as well as the methods of detection and control of variables may behave differently.

Heat and mass transfer - The study of heat and mass transfer phenomena in bioprocesses is substantial to allow the encounter of all the transformation reagents (culture media, cells and cofactors) under the same conditions (temperature, $\mathrm{pH}$ and concentration of substances). Consequently, a mixing method is required in order to guarantee a proper distribution of reagents and parameters in the fermentative broth, playing an important role on the final production and also on the precision of measured data (Schäpper et al., 2009). Owing to the small Re number applied, a laminar condition is predominant over turbulence within microbioreactors, making it more difficult to achieve good mixing as the working volume decreases (Purcell, 1977). Even more, different strains might have different sensitivities to mechanical movements, thus characterising optimal mixing as a trade-off between homogeneity and cell shear stress (Schäpper et al., 2009).

Mixing methods in microbioreactors can be divided into active and passive. Active methods usually use either free floating or supported impellers inserted into the microbioreactor (Puskeiler et al., 2005; Tan et al., 2015). This application requires extra caution when designing the bioreactor because the appearance of dead zones might reduce the mixing profile over the fermentation chamber. Zhang et al. (2006) constructed a microbioreactor with good mixing profiles using a magnetic stir bar of stirring velocity up to $800 \mathrm{rpm}$. Such a device could reach $K_{L} a$ values up to $75 \mathrm{~h}^{-1}$. Nevertheless, active mixing without the assembling of moving parts has been recently studied. For example, Kirk et al. (2016) have developed a microbioreactor capable of promoting active mixing, the mechanism of which relies on the presence of a single syringe promoting an oscillating jet by recirculating the culture medium in and out of the fermentative chamber. $K_{L} a$ values in this device reached around $170 \mathrm{~h}^{-1}$.

Passive mixing might be achieved by molecular diffusion and chaotic advection by applying the fermentation broth under a flow, which might be disturbed by bifurcations and convergences, further enhanced by the placement of obstacles in the flow streams (Schäpper et al., 2009). Stroock et al. (2002) described a passive mixing method that relies on a transverse swirling fluidic motion caused by a staggered herringbone mixer.

Another relevant parameter concerning heat and mass transfer in biotechnological processes is aeration. Aerobic fermentations are a great parcel of industrial bioprocesses and require a proper level of available oxygen for respiration purposes (Schmidell et al., 2001). The material used for constructing microbioreactors may influence the capacity of aeration inside the fermentative chamber. PDMS, as discussed before, is permeable to gases and may be used for the construction of thin membranes able to promote the diffusion of oxygen and other gases (offgases) produced in the fermentation process without compromising the purity of the broth with contaminant agents (Zanzotto et al., 2004; Szita et al., 2005; Lee et al., 2006; Zhang et al., 2006; Schäpper et al., 2009). Not only for aeration purposes, Schäpper et al. (2010) used PDMS membranes for the application of $\mathrm{CO}_{2}$ and $\mathrm{NH}_{3}$ gases with the aim of controlling $\mathrm{pH}$ levels. Nevertheless, PDMS membranes are not selective to gases, allowing the diffusion of water vapour out of the fermentative chamber (Berthier et al., 2008; Schäpper et al., 2009). Solutions may include reducing evaporation either by placing the whole device within a humidified chamber or by connecting a water reservoir above the microbioreactor level to allow passive replenishment of water (Szita et al., 2005; Schäpper et al., 2009).

Therefore, oxygen availability in the fermentative broth might be enhanced by combining both mixing and aeration strategies. In this regard, Kirk and Szita (2013) have revised the phenomenon of oxygen transfer and $K_{L} a$ values under miniaturized bioreactors. As most of the mini-scale systems display analogous $K_{L} a$ values, they can be considered proper scaleddown tools of mainstream bench-scale bioreactors for bioprocess development, with the additional advantage of offering more integration with online detectors and controlling tools, also capable of accelerating data acquisition with reduced costs.

Detection and control methods - Biological systems are extremely complex, being governed by the interactions between environmental conditions and genetic responses. The study and development of sophisticated bioprocesses require the capacity of analysing such interactions. Therefore, the detection and control of significant conditions and parameters are crucial for the performance of bioprocess experiments.

It is important to remember that microtechnology is a very sensitive approach and a small change in the sample volume might interfere in the final results. Therefore, there is a necessity to apply non-invasive methods that do not require sample collection or 
changes in the working volume applied. A good example is the implementation of optical detectors (optodes), which can cover a wide number of variables and generally have a good range of detection limits (Nge et al., 2013).

Schäpper et al. (2009) reviewed the strategies and instruments commonly applied to microbioreactors for the sensing and control of important parameters, which will be briefly discussed. In such a scenario, key variables may consist of temperature, $\mathrm{pH}$, cellular density and dissolved oxygen.

Temperature is a variable relatively easy to be measured and is generally sensed by resistance temperature detectors (RTDs) or even thermocouples (Schäpper et al., 2009). RTDs are tools capable of measuring temperature via their resistance variation. On the other hand, thermocouples are sensors composed of two different metals welded at one end forming a junction, where a potential difference is generated due to temperature variation. Although thermocouples have a fast time response to variations, RTDs are more accurate and prevail in stability for much longer periods of time. Furthermore, RTDs are found on the market at very cheap prices and can be easily integrated within a microbioreactor structure (Schäpper et al., 2009). Controlling temperature, on the other hand, might be complicated regarding the sensitivity offered by the micro scale. As the proportions of surface area to working volume are higher in such circumstances, heat might be quickly exchanged between microbioreactors and the environment (Schäpper et al., 2009). Furthermore, temperature homogeneity throughout the microdevice might be a concern when materials of low thermal conductivity, such as PMMA and PDMS, are adopted. A simple solution regarding temperature control might be placing microdevices into incubators or water baths (Schäpper et al., 2009). Even though such approaches would allow the parallelization of several devices at the same place with the same temperature accurately conditioned, experiments aiming to study the effects of different temperature levels would be less practical. A feasible and cheap alternative able to work around such drawbacks would be implementing microheaters within the microbioreactor chamber (Schäpper et al., 2009).

Sensing and controlling $\mathrm{pH}$ might be a challenging task since conventional methods rely on voluminous probes and chemical additions, directly interfering in the total working volume. Within the microscale, $\mathrm{pH}$ is generally measured via ion-sensitive field-effect transistor chips (ISFET) and optodes. ISFET detectors rely on current changes within a transistor based on the variation of ion concentrations (e.g., $\mathrm{H}^{+}$and $\mathrm{OH}^{-}$) (Bergveld, 2003; Lee et al., 2009), while optodes measure $\mathrm{pH}$ through either fluorescence lifetime measurements or fluorescence intensity. Even though ISFET detectors are able to work on a wider range of $\mathrm{pH}$ with linear responses (voltage to $\mathrm{pH}$ units), higher accuracy and faster response time, optodes tend to be a favourite option for $\mathrm{pH}$ measurement within microbioreactors due to their ease of integration and non-invasive nature (Schäpper et al., 2009). Moreover, unlike optodes, ISFET detectors require an electrode for reference, making the design of microbioreactors more complex as ISFET detectors would need to be reusable regarding cost purposes (Schäpper et al., 2009). For $\mathrm{pH}$ control, the small nature of the micro scale imposes a key limitation. Alternatives may consist of implementing buffers in the composition of the medium or directly injecting strong basic or acid chemicals in the culture (Schäpper et al., 2009). Both approaches are very limited: buffers generally work in a specific $\mathrm{pH}$ range and are limited to a certain number of ions; and the injection of additives directly interferes in the total working volume, which is a critical issue under the micro scale, with an extra drawback that good mixing levels are necessary for the homogeneity of the culture (Schäpper et al., 2009). A promising solution might consist of applying either acid or basic gases for $\mathrm{pH}$ control (Isett et al., 2007). Nevertheless, bubbles might appear as the gas diffuses through the aqueous phase, an unwanted scenario in microbioreactors (Schäpper et al., 2009).

The concentration of cells, or cellular density, can be easily monitored by the traditional method using absorbance measurements as dictated by the LambertBeer law. The spectrophotometry technique has been feasibly applied on the micro scale via optical probes, using light-emitting diodes (LED) as a light source and a photodetector as a detection tool. An important concern regarding such an approach might be the positioning of the light emitter and collector (Schäpper et al., 2009). As microbrioreactors tend to be designed as a flat structure, the vertical positioning of detectors would imply the travel of light within the cultivation chamber via a short path length, a good option for accurate monitoring of higher cell densities (Schäpper et al., 2009). On the other hand, by positioning the apparatus over a horizontal reading frame, meaning less intense illumination, lower cellular densities might be more accurately measured (Schäpper et al., 2009). Therefore, when changing the position of the spectrophotometric apparatus in disposable microbioreactors, new calibrations are necessary. Additional concerns include the alignment of fibres to avoid transmitted light loss, absence of interfering agents such as mixing beads or bubbles to avoid light dispersion and external light interference (Schäpper et al., 2009). 
The detection and control of dissolved oxygen (DO) levels is crucial for aerated processes. This variable can be easily measured by optodes, which rely on the quenching offluorescence by oxygen in order to measure its concentration in the fermentative broth (Schäpper et al., 2009). This method of detection is non-invasive, cheap, easy to be implemented and might increase its sensitivity as long as oxygen concentration decreases, perfectly fitting the characteristics of fermentation processes and microtechnology requisites (Schäpper et al., 2009). Nevertheless, controlling DO in the broth of microbioreactors might be very challenging as bubbles are not desirable and mixing systems are not always accessible on the micro scale. Feasible solutions might consist of diffusing oxygen through gas-permeable membranes that allow the diffusion of $\mathrm{O}_{2}$ from aerating systems or gas chambers placed over the top of the cultivation chamber, increasing the diffusive rate of the gas phase into the liquid phase (Schäpper et al., 2009). Furthermore, enhancing the level of dissolved oxygen within microbioreactors might be achieved by increasing the content of $\mathrm{O}_{2}$ in the gas phase and, when possible, applying a mixing system able to provide good levels of homogeneity (Schäpper et al., 2009).

\section{FINAL CONSIDERATIONS}

Microbioreactors have been successfully developed and applied to fermentative bioprocesses over the past years. Several works have proven beautifully the feasibility of applying this technology with the aim of studying general bioprocess kinetics. Consequently, there has been increasing acceptance and adoption of microbioreactors and microscale techniques on the field of bioprocesses since 2004 .

In current society, where the bio-based economy is growing consistently along with a shifting of chemical processes to the biotechnology route, the development and optimization of bioprocesses is crucial for the establishment of a solid industry capable of overcoming recurrent problems and drawbacks. Within this background, microbioreactors consist of an interesting strategy for the screening of parameters. When correctly established, they are able to provide a massive quantity of accurate data in a small period of time, in a feasible and cheap manner.

Even though such technology may look attractive and even be the solution for current issues, establishing a centre fully equipped with proper facilities for the development of microbioreactors requires a substantial investment. Allied with the apprehension of scientists and the relatively poor number of studies concerning the contributions that micro-scale technology may offer to industrial biotechnology, this strategy has yet to achieve popularity in order to gain a considerable space in bioprocess laboratories. Current limitations of microbioreactors as tools for biotechnology process studies still rely on the sensing and detecting methods of specific variables. Most applications are generally limited to the detection and control of temperature, cell density, $\mathrm{pH}$ and gas concentration within a relatively limited range. In this manner, complex biotechnology processes may face barriers when adopting such technology, requiring feasible and inexpensive sensors with higher levels of versatility and sophistication. Furthermore, scalability is still the reason for drawbacks. The nature of the small dimensions is different from the conventional bench scale and may not be directly compared, especially when scaling up results acquired with microdevices.

Considering this scenario, this study finally concludes that there is much to explore within the micro-scale world for bioprocesses and industrial biotechnology. This approach has a great potential and is still in its infancy. Therefore, there is much to be discovered and developed, making microbioreactor technology an exciting field of study. Furthermore, because of its multi-, inter- and transdisciplinary nature, it requires the collaborative work of various researchers from different sciences for the development of feasible, user friendly, and maybe even revolutionary tools capable of reducing costs and the time spent during the engineering of industrial bioprocesses.

\section{REFERENCES}

Abeille, F.; Mittler, F.; Obeid, P.; Huet, M.; Kermarrec, F.; Dolega, M.E.; Navarro, F.; Pouteau, P.; Icard, B.; Gidrol, X.; Agache, V.; and Picollet-D'hahan, N., Continuous microcarrier-based cell culture in a benchtop microfluidic bioreactor. Lab on a Chip, 14, 3510-3518 (2014).

Addae-Mensah, K.; Wang, Z.; Parsa, H.; Chin, S.; Laksanasopin, T.; and Sia, S., Fundamentals of Microfluidics Devices. Microfluid Devices Nanotechnol. Hoboken: John Wiley \& Sons Inc; p. 1-38 (2010).

Atencia, J.; Cooksey, G.A.; and Locascio, L.E., A robust diffusion-based gradient generator for dynamic cell assays. Lab on a Chip, 12, 309-316 (2012).

Au, A.K.; Huynh, W.; Horowitz, L.F.; and Folch, A., 3D-Printed Microfluidics. Angewandte Chemie Int. Ed.., 55, 3862-3881 (2016).

$\mathrm{Au}, \mathrm{S} . \mathrm{H} . ;$ Shih, S.C.C.; and Wheeler, A.R., Integrated microbioreactor for culture and analysis of bacteria, algae and yeast. Biomedical Microdevices, 13(1), 41-50 (2011). 
Baranek, S.; Bezler, A.; Adamczyk, C.; Gönczy, P.; and Renaud, P., Temperature Gradient Stimulation for Cell Division. Molecular Cell, 1(1), 190-192 (2010).

Baret, J-C., Surfactants in droplet-based microfluidics. Lab on a Chip, 12, 422-433 (2012).

Baroud, C.N.; Gallaire, F.; and Dangla, R., Dynamics of microfluidic droplets. Lab on a Chip, 10, 20322045 (2010).

Basova, E.Y.; and Foret, F., Droplet microfluidics in (bio)chemical analysis. Analyst, 140, 22-38 (2014).

Beebe, D.J.; Mensing, G.A.; and Walker, G.M., Physics and applications of microfluidics in biology. Annual Reviews of Biomedical Engineering, 4, 261-286 (2002).

Bergveld, P., Thirty years of ISFETOLOGY. Sensors and Actuators B: Chemical, 88, 1-20 (2003).

Berthier, E.; Warrick. J.; Yu, H.; and Beebe, D.J., Managing evaporation for more robust microscale assays: Part 1. Volume loss in high throughput assays. Lab on a Chip, 8, 852-859 (2008).

Berthier, J.; and Silberzan, P., Microfluidics for Biotechnology. 2nd Edition. Norwood: Artech House (2009).

Björnmalm, M.; Yan, Y.; and Caruso, F., Engineering and evaluating drug delivery particles in microfluidic devices. Journal of Controlled Release, 190, 139-149 (2014).

Blesken, C.; Olfers, T.; Grimm, A.; and Frische, N., The microfluidic bioreactor for a new era of bioprocess development. Engineering in Life Sciences, 16, 190-193 (2016).

Breslauer, D.N.; Lee, P.J.; and Lee, L.P., Microfluidicsbased systems biology. Molecular Biosystems, 2, 97-112 (2006).

Chang, W.C.; Lee, L.P.; and Liepmann, D., Biomimetic technique for adhesion-based collection and separation of cells in a microfluidic channel. Lab on a Chip, 5, 64-73 (2005).

Chiang, H-J.; Yeh, S-L.; Peng, C-C.; Liao, W-H.; and Tung, Y-C., Polydimethylsiloxane-polycarbonate Microfluidic Devices for Cell Migration Studies Under Perpendicular Chemical and Oxygen Gradients. Journal of Visualized Experiments, 23(120), e55292-e55292 (2017).

Chin, D.; Laksanasopin, T.; Cheung, Y.; Steinmiller, D.; Linder, V.; Parsa, H.; Wang, J.; Moore, H.; Rouse, R.; Umviligihozo, G.; Karita, E.; Mwambarangwe, L.; Braunstein, S.; van de Wijgert, J.; Sahabo, R.; Justman, J.; El-Sadr, W.; and Sia, S., Microfluidicsbased diagnostics of infectious diseases in the developing world. Nature Medicine, 17, 10151019 (2011).

Chung, B.G.; Flanagan, L.A.; Rhee, S.W.; Schwartz, P.H.; Lee, A.P.; Monuki, E.S.; and Jeon, N.L., Human neural stem cell growth and differentiation in a gradient-generating microfluidic device. Lab on a Chip, 5:401-406 (2005).
Conchouso, D.; Castro, D.; Khan, S.A.; and Foulds I.G., Three-dimensional parallelization of microfluidic droplet generators for a litre per hour volume production of single emulsions. Lab on a Chip, 14, 3011-20 (2014).

Cooksey, G.A.; Sip, C.G.; and Folch, A., A multipurpose microfluidic perfusion system with combinatorial choice of inputs, mixtures, gradient patterns, and flow rates. Lab on a Chip, 9, 417-26 (2009).

de Raad, M.; Fischer, C.R.; and Northen T,R., Highthroughput platforms for metabolomics. Current Opinion in Chemical Biology, 30, 7-13 (2016).

Delamarche, E.; Schmid, H.; Bietsch, A.; Michel, B.; and Biebuyck, H., Microfluidic Networks for Chemical Patterning of Substrates. Journal of the American Chemical Society ,7863, 1-9 (1998).

DeMello, A.J., Control and detection of chemical reactions in microfluidic systems. Nature, 442, 394-402 (2006).

Di Carlo, D.; Wu, L.Y.; and Lee, L.P., Dynamic single cell culture array. Lab on a Chip, 6, 1445-1449 (2006).

Dittrich, P.S.; and Manz, A., Lab-on-a-chip: microfluidics in drug discovery. Nature Reviews Drug Discovery, ,5, 210-218 (2006).

El-Ali, J.; Sorger, P.K.; and Jensen, K.F., Cells on chips. Nature, 442, 403-411 (2006).

Eriksson, E.; Scrimgeour, J.; Granéli, A.; Ramser, K.; Wellander, R.; Enger, J.; Hanstorp, D.; and Goksör, M., Optical manipulation and microfluidics for studies of single cell dynamics. Journal of Optics A: Pure and Applied Optics, 9, S113-S121 (2007).

Fair, R.B.; Khlystov, A.; Tailor, T.D.; Ivanov, V.; Evans, R.D.; Pollack, M.G.; Griffin, P.B.; and Zhou, J., Chemical and Biological Applications of Digital-Microfluidic Devices. IEEE Design and Test of Computers, 24, 10-24 (2007).

Fernandes, P.; Carvalho, F.; and Marques, M.P.C., Miniaturization in biotechnology: speeding up the development of bioprocesses. Recent Patents on Biotechnology, 5, 160-73 (2011).

Gao, A.; Yang, X.; Tong, J.; Zhou, L.; Wang, Y.; Zhao, J.; Mao, H.; and Li, T., Multiplexed detection oflung cancer biomarkers in patients serum with CMOScompatible silicon nanowire arrays. Biosensors and Bioelectronics, 91, 482-488 (2017).

Grünberger, A.; Wiechert, W.; and Kohlheyer, D., Single-cell microfluidics: Opportunity for bioprocess development. Current Opinion in Biotechnology, 29, 15-23 (2014).

He, Y.; Wu, Y.; Fu, J.Z.; Gao, Q.; and Qiu, J.J., Developments of 3D Printing Microfluidics and Applications in Chemistry and Biology: a Review. Electroanalysis, 28, 1658-1678 (2016). 
Hegab, H.M.; ElMekawy, A.; and Stakenborg, T., Review of microfluidic microbioreactor technology for high-throughput submerged microbiological cultivation. Biomicrofluidics, 7(2), 21502 (2013).

Ho, C.M.B.; Ng, S.H.; Li, K.H.H.; and Yoon, Y-J., 3D printed microfluidics for biological applications. Lab on a Chip, 15, 3627-3637 (2015).

Holtze, C., Large-scale droplet production in microfluidic devices - an industrial perspective. Journal of Physics D Applied Physics, 46(11), 114008 (2013).

Houshmand, M.; Soleimani, M.; Atashi, A.; Saglio, G.; Abdollahi, M.; and Nikougoftar Zarif, M., Mimicking the Acute myeloid leukemia niche for molecular study and drug screening. Tissue Engineering Part C: Methods, 23(2), 72-85, (2017).

Huebner, A.; Sharma, S.; Srisa-Art, M.; Hollfelder, F.; Edel, J.B.; and Demello, A.J., Microdroplets: a sea of applications? Lab on a Chip, 8, 1244-1254 (2008).

Hung, P.J.; Lee, P.J.; Sabounchi, P.; Lin, R.; and Lee, L.P., Continuous perfusion microfluidic cell culture array for high-throughput cell-based assays. Biotechnology and Bioengineering, 89(1), 1-8 (2005).

Huo, W.; Gao, Y.; Zhang, L.; Shi, S.; and Gao, Y., A Novel High-Sensitivity Cardiac Multibiomarker Detection System Based on Microfluidic Chip and GMR Sensors. IEEE Transactions on Magnetics, 51, 18-21 (2015).

Isett, K.; George, H.; Herber, W.; and Amanullah, A., Twenty-four-well plate miniature bioreactor high-throughput system: Assessment for microbial cultivations. Biotechnology and Bioengineering, 98, 1017-1028 (2007).

Ismagilov, R.F.; Ng, J.M.K.; Kenis, P.J.A.; and Whitesides, G.M., Microfluidic arrays of fluidfluid diffusional contacts as detection elements and combinatorial tools. Analytical Chemistry, 73, 5207-5213 (2001).

Jacques, P.; Béchet, M.; Bigan, M.; Caly, D.; Chataigné, G.; Coutte, F.; Flahaut, C.; Heuson, E.; Leclère, V.; Lecouturier, D.; Phalip, V.; Ravallec, R.; Dhulster, P.; and Froidevaux, R., High-throughput strategies for the discovery and engineering of enzymes for biocatalysis. Bioprocess and Biosystems Engineering, 40, 161-180 (2017).

Jeon, N.L.; Dertinger, S.K.W.; Chiu, D.T.; Choi, I.S.; Stroock, A.D.; and Whitesides, G.M., Generation of Solution and Surface Gradients using Microfluidic Systems. Langmuir, 16, 8311-8316 (2000).

Johnson-Chavarria, E.M.; Agrawal, U.; Tanyeri, M.; Kuhlman, T.E.; and Schroeder, C.M., Automated single cell microbioreactor for monitoring intracellular dynamics and cell growth in free solution. Lab on a Chip, ,14, 1-10 (2014).
Keenan, T.M.; Hsu, C.H.; and Folch, A., Microfluidic "jets" for generating steady-state gradients of soluble molecules on open surfaces. Applied Physics Letters, 89, 2012-2015 (2006).

Kim, S.; Kim, H.J.; and Jeon, N.L., Biological applications of microfluidic gradient devices. Integrative Biology, 2, 584-603 (2010).

Kim, T.H.; Saadatpour, A.; Guo, G.; Saxena, M.; Cavazza, A.; Desai, N.; Jadhav, U.; Jiang, L.; Rivera, M.N.; Orkin, S.H.; Yuan, G.C.; and Shivdasani, R.A., Single-Cell Transcript Profiles Reveal Multilineage Priming in Early Progenitors Derived from Lgr5+ Intestinal Stem Cells. Cell Reports, 16, 2053-2060 (2016).

Kirk, T.V.; Marques, M.P.C.; Radhakrishnan, A.N.P.; and Szita, N., Quantification of the oxygen uptake rate in a dissolved oxygen controlled oscillating jet-driven microbioreactor. Journal of Chemical Technology and Biotechnology, ;91, 823-831 (2016).

Kirk, T.V.; and Szita, N., Oxygen transfer characteristics of miniaturized bioreactor systems. Biotechnology and Bioengineering 110, 1005-1019 (2013).

Kostov, Y.; Harms, P.; Randers-Eichhorn, L.; and Rao, G., Low-cost microbioreactor for high throughput bioprocessing. Biotechnology and Bioengineering, 72, 346-352 (2001).

Kotz, F.; Arnold, K.; Wagner, S.; Bauer, W.; Keller, N.; Nargang, T.M.; Helmer, D.; and Rapp, B.E., Liquid PMMA:AHigh Resolution Polymethylmethacrylate Negative Photoresist as Enabling Material for Direct Printing of Microfluidic Chips. Advanced Engineering Materials, 1700699, 1-5 (2017).

Krull, R.; and Peterat, G., Analysis of reaction kinetics during chemostat cultivation of Saccharomyces cerevisiae using a multiphase microreactor. Biochemical Engineering Journal, 105, 220-229 (2016).

Lam, E.T.; Hastie, A.; Lin, C.; Ehrlich, D.; Das, S.K.; Austin, M.D.; Deshpande, P.; Cao, H.; Nagarajan, N.; Xiao, M.; and Kwok, P.Y., Genome mapping on nanochannel arrays for structural variation analysis and sequence assembly. Nat Biotechnol. Nature, 30, 771-776 (2012).

Lee, C.S.; Kim, S.K., and Kim, M., Ion-sensitive fieldeffect transistor for biological sensing. Sensors, 9, 7111-7131 (2009).

Lee, P.J.; Hung, P.J.; Rao, V.M.; Lee, and L.P., Nanoliter scale microbioreactor array for quantitative cell biology. Biotechnology and Bioengineering, 94, 5-14 (2006).

Li, M.; Shi, Z.Z.; Fang, C.; Gao, A.X.; Li, C.M.; and Yu, L., Versatile microfluidic complement fixation test for disease biomarker detection. Analytica Chimica Acta, 916, 67-76 (2016). 
Li, Y.; Chen, D.; Zhang, Y.; Liu, C.; Chen, P.; Wang, Y.; Feng, X.; Du, W.; and Liu, B.F., High-throughput single cell multidrug resistance analysis with multifunctional gradients-customizing microfluidic device. Sensors and Actuators B: Chemical, 225, 563-571 (2016).

Livak-Dahl, E.; Sinn, I.; Burns, M., Microfluidic chemical analysis systems. Annual Review of Chemical and Biomolecular Engineering, 2, 32553 (2011).

Lucchetta, E.M.; Lee, J.H.; Fu, L.A.; Patel, N.H; and Ismagilov, R.F., Dynamics of Drosophila embryonic patterning network perturbed in space and time using microfluidics. Nature, 434, 11341138 (2005).

Luo, Y.; Liu, C.; Qu, Y.; and Fang, N., Towards singlecell analysis for pharmacokinetics. Bioanalysis, 4, 453-63 (2012).

Macosko, E.Z.; Basu, A.; Satija, R.; Nemesh, J.; Shekhar, K.; Goldman, M.; Tirosh, I.; Bialas, A.R.; Kamitaki, N.; Martersteck, E.M.; Trombetta, J.J.; Weitz, D.A.; Sanes, J.R.; Shalek, A.K.; Regev, A.; and McCarroll, S.A., Highly parallel genomewide expression profiling of individual cells using nanoliter droplets. Cell, 161, 1202-1214 (2015).

Maglica, Ž.; Özdemir, E.; and McKinney, J.D., SingleCell Tracking Reveals Antibiotic-Induced Changes in Mycobacterial Energy Metabolism. MBio, 6(1), e02236-14 (2015).

Maharbiz, M.M.; Holtz,W.J.; Howe, R.T.; and Keasling, J.D., Microbioreactor Arrays with Parametric Control for High-Throughput Experimentation. Biotechnology and Bioengineering, 85, 376-381 (2004).

Maisch, J.; Kreppenhofer, K.; Büchler, S.; Merle, C.; Sobich, S.; Görling, B.; Luy, B.; Ahrens, R.; Guber, A.E.; and Nick, P., Time-resolved NMR metabolomics of plant cells based on a microfluidic chip. Journal of Plant Physiology, 200, 28-34 (2016).

Marcus, J.S.; Anderson, W.F.; and Quake, S.R., Microfluidic single-cell mRNA isolation and analysis. Analytical Chemistry, 78, 3084-3089 (2006).

Marques, M.P.C.; and Fernandes, P., Microfluidic devices: Useful tools for bioprocess intensification. Molecules, 16, 8368-8401 (2011).

McDonald, J.C.; and Whitesides, G.M., Poly(dimethylsiloxane) as a material for fabricating microfluidic devices. Accounts of Chemical Research, 35, 491-499 (2002).

Micheletti, M.; and Lye, G.J., Microscale bioprocess optimisation. Current Opinion in Biotechnology, 17, 611-618 (2006).
Mosadegh, B.; Huango, C.; Park, J.W.; Shin, H.S.; Chung, B.G.; Hwang, S.K.; Lee, K.H.; Kim, H.J.; Brody, J.; and Jeon, N.L., Generation of stable complex gradients across two-dimensional surfaces and three-dimensional gels. Langmuir, 23, 1091010912 (2007).

Nge, P.N.; Rogers, C.I.; and Woolley, A.T., Advances in Microfluidic Materials, Functions, Integration, and Applications. Chemical Reviews, 113(4), 2550-2583 (2013).

Oliveira, A.F.; Pessoa, A.C.S.N.; Bastos, R.G.; and de la Torre, L.G., Microfluidic tools toward industrial biotechnology. Biotechnology Progress, 32, 13721389 (2016).

Pan, J.; Stephenson, A.L.; Kazamia, E.; Huck, W.T.S.; Dennis, J.S.; Smith,A.G.; and Abell, C., Quantitative tracking of the growth of individual algal cells in microdroplet compartments. Integrative Biology, 3, 1043-1051 (2011).

Patou, F.; Dimaki, M.; Svendsen, W.E.; Kjaegaard, K.; and Madsen, J., A smart mobile lab-on-chip-based medical diagnostics system architecture designed for evolvability. 2015 Euromicro Conference on Digital System Design, 390-398 (2015).

Pihl, J.; Sinclair, J.; Sahlin, E.; Karlsson, M.; Petterson, F.; Olofsson, J.; and Orwar, O., Microfluidic gradient-generating device for pharmacological profiling. Analytical Chemistry, 77, 3897-3903 (2005)

Purcell, E.M., Life at low Reynolds number. American Journal of Physics, 45, 3-11 (1977).

Puskeiler, R.; Kaufmann, K.; and Weuster-Botz, D., Development, parallelization, and automation of a gas-inducing milliliter-scale bioreactor for high-throughput bioprocess design (HTBD). Biotechnology and Bioengineering, 89, 512-523 (2005).

Qin, D.; Xia, Y.; and Whitesides, G.M., Soft lithography for micro- and nanoscale patterning. Nature Protocols, 5, 491-502 (2010).

Sackmann, E.K.; Fulton, A.L.; and Beebe, D.J., The present and future role of microfluidics in biomedical research. Nature 507, 181-189 (2014).

Saka, Y.; MacPherson, M.; and Giuraniuc, C.V., Generation and precise control of dynamic biochemical gradients for cellular assays. Physica A: Statistical Mechanics and its Applications, 470, 132-145 (2017).

Sanders, G.H.W.; and Manz, A., Chip-based microsystems for genomic and proteomic analysis. TrAC - Trends in Analytical Chemistry, 19, 364378 (2000).

Schäpper, D.; Alam, M.N.H.Z.; Szita, N.; Eliasson Lantz, A.; and Gernaey, K.V., Application of microbioreactors in fermentation process development: A review. Analytical and Bioanalytical Chemistry, 395, 679-695 (2009). 
Schäpper, D.; Stocks, S.M.; Szita, N.; Lantz, A.E.; and Gernaey, K.V., Development of a single-use microbioreactor for cultivation of microorganisms. Chemical Engineering Journal, 160, 891-898 (2010).

Schmidell, W.; Lima, U.; Aquarone, E.; and Borzani, W., Biotecnologia Industrial. São Paulo: Edgard Blücher Ltda (2001).

Shalek, A.K.; Satija, R.; Shuga, J.; Trombetta, J.J.; Gennert, D.; Lu, D.; Chen, P.; Gertner, R.S.; Gaublomme, J.T.; Yosef, N.; Schwartz, S.; Fowler, B.; Weaver, S.; Wang, J.; Wang, X.; Ding, R.; Raychowdhury, R.; Friedman, N.; Hacohen, N.; Park, H.; May, A.P.; and Regev, A., Single-cell RNA-seq reveals dynamic paracrine control of cellular variation. Nature, 510, 363-369 (2014).

Sista, R.; Hua, Z.; Thwar, P.; Sudarsan, A.; Srinivasan, V.; Eckhardt, A.; Pollack, M.; and Pamula, V., Development of a digital microfluidic platform for point of care testing. Lab on a Chip, 8, 2091-2104 (2008).

Sjostrom, S.L.; Bai, Y.; Huang, M.; Liu, Z.; Nielsen, J.; Joensson, H.N.; and Andersson Svahn, H., High-throughput screening for industrial enzyme production hosts by droplet microfluidics. Lab on a Chip, 14, 806-813 (2014).

Squires, T.M.; and Quake, S.R., Microfluidics: Fluid physics at the nanoliter scale. Reviews of Modern Physics, 77, 977-1026 (2005).

Stott, S.L.; Hsu, C-H.; Tsukrov, D.I.; Yu, M.; Miyamoto, D.T.; Waltman, B.A.; Rothenberg, S.M.; Shah, A.M.; Smas, M.E.; Korir, G.K.; Floyd, F.P.; Gilman, A.J.; Lord, J.B.; Winokur, D.; Springer, S.; Irimia, D.; Nagrath, S.; Sequist, L.V.; Lee, R.J.; Isselbacher, K.J.; Maheswaran, S.; Haber, D.A.; and Toner, M., Isolation of circulating tumor cells using a microvortex-generating herringbone-chip. Proceedings of the National Academy of Sciences of the United States of America October, 107(43), 18392-18397 (2010).

Stroock, A.; Dertinger, S.; Ajdari, A.; Mezi. I.; Stone, H.; and Whitesides, G., Chaotic Mixer for Microchannels. Science, 295, 647-651 (2002).

Sung, J.H.; and Shuler, M.L., A micro cell culture analog (microCCA) with 3-D hydrogel culture of multiple cell lines to assess metabolism-dependent cytotoxicity of anti-cancer drugs. Lab on a Chip, 9, 1385-1394 (2009).

Szita, N.; Boccazzi, P.; Zhang, Z.; Boyle, P.; Sinskey, A.J.; and Jensen, K.F., Development of a multiplexed microbioreactor system for highthroughput bioprocessing. Lab on a Chip, 5, 819826 (2005).

Szita, N.; Polizzi, K.; Jaccard, N.; Baganz, F., Microfluidic approaches for systems and synthetic biology. Current Opinion in Biotechnology, 21, 517-523 (2010)
Tan, C.K.; Davies, M.J.; Mccluskey, D.K.; Munro, I.R.; Nweke, M.C.; Tracey, M.C.; and Szita, N., Electromagnetic stirring in a microbioreactor with non-conventional chamber morphology and implementation of multiplexed mixing. Journal of Chemical Technology and Biotechnology, 90, 1927-1936 (2015).

Tay, S.; Hughey, J.J.; Lee, T.K.; Lipniacki, T.; Quake, S.R.; and Covert, M.W., Single-cell NF-kappaB dynamics reveal digital activation and analogue information processing. Nature, 466, 267-71 (2010).

Teh, S-Y.; Lin, R.; Hung, L-H.; and Lee, A.P., Droplet microfluidics. Lab on a Chip, 8, 198-220 (2008).

Terry, S.C.; Herman, J.H.; and Angell, J.B., A gas chromatographic air analyzer fabricated on a silicon wafer. IEEE Transactions on Electron Devices, 26, 1880-1886 (1979).

Toh, A.G.G.; Wang, Z.P.; Yang, C.; and Nguyen, N.T., Engineering microfluidic concentration gradient generators for biological applications. Microfluidics and Nanofluidics, 16, 1-18 (2014).

Toriello, N.M.; Douglas, E.S.; Thaitrong, N.; Hsiao, S.C.; Francis, M.B.; Bertozzi, C.R.; and Mathies, R.A., Integrated microfluidic bioprocessor for single-cell gene expression analysis. Proceedings of the National Academy of Sciences of the United Sstates of Aamerica, 105, 20173-20178 (2008).

Tseng, P.; Murray, C.; Kim, D.; and Di Carlo, D., Research highlights: printing the future of microfabrication. Lab on a Chip, 14, 1491-1495 (2014).

Wang, D.; and Bodovitz, S., Single cell analysis: The new frontier in "omics". Trends in Biotechnology, 28, 281-290 (2010).

Wang, J.; and Yang, F., Emerging single-cell technologies for functional proteomics in oncology. Expert Review of Proteomics, 13 805-815 (2016).

Weibel, D.B.; Diluzio, W.R.; and Whitesides, G.M., Microfabrication meets microbiology. Nature Reviews Microbiology, 5, 209-18 (2007).

Weibel, D.B.; and Whitesides, G.M., Applications of microfluidics in chemical biology. Current Opinion in Chemical Biology, 10, 584-591 (2006).

Whitesides, G.M., The origins and the future of microfluidics. Nature, 442 368-73 (2006).

Wlodkowic, D.; Faley, S.; Zagnoni, M.; Wikswo, J.P.; and Cooper, J.M., Microfluidic Single-Cell Array Cytometry for the Analysis of Tumor Apoptosis. Analytical Chemistry, 81, 5517-5523 (2009).

Wolfe, D.B.; Qin, D.; and Whitesides, G.M., Rapid Prototyping of Microstructures by Soft Lithography for Biotechnology. Methods of Molecular Biology, 583, 81-107 (2010).

Wolfram, C.J.; Rubloff, G.W.; and Luo, X., Perspectives in flow-based microfluidic gradient generators for characterizing bacterial chemotaxis. Biomicrofluidics, 10, 061301 (2016). 
Xia, Y.; and Whitesides, G.M., Soft Lithography. Angewandte Chemie International Edition, 37, 550-575 (1998).

Xie, X.; Cheng, Z.; Xu, Y.; Liu, R.; Li, Q.; and Cheng, J., A sheath-less electric impedance microflow cytometry device for rapid label-free cell classification and viability testing. Analytical Methods, 9, 1201-1212 (2017).

Xu, Z.; Huang, X.; Wang, P.; Wang, H.; and Weitz, D.A., Optimization and development of a universal flow-based microfluidic gradient generator. Microfluidics and Nanofluidics, 20, 1-10 (2016).

Yang, W.; Lee, K.K.; and Choi, S., A laminar-flow based microbial fuel cell array. Sensors and Actuators B: Chemical, 243, 292-297 (2017).

Yu, M.; Bardia, A.; Aceto, N.; Bersani, F.; Madden, M.W.; Donaldson, M.C.; Desai, R.; Zhu, H.; Comaills, V.; Zheng, Z.; Wittner, B.S.; Stojanov, P.; Brachtel, E.; Sgroi, D.; Kapur, R.; Shioda, T.; Ting, D.T.; Ramaswamy, S.; Getz, G.; Iafrate, A.J.; Benes, C.; Toner, M.; Maheswaran, S.; and Haber, D.A., Cancer therapy. Ex vivo Culture of
Circulating Breast Tumor Cells for Individualized Testing of Drug Susceptibility. Science, 345, 216220 (2014).

Zanzotto, A.; Szita, N.; Boccazzi, P.; Lessard, P.; Sinskey, A.J.; and Jensen, K.F., Membrane-aerated microbioreactor for high-throughput bioprocessing. Biotechnology and Bioengineering, 87, 243-254 (2004).

Zhang, Z.; Szita, N.; Boccazzi, P.; Sinskey, A.J.; and Jensen, K.F., A well-mixed, polymerbased microbioreactor with integrated optical measurements. Biotechnology and Bioengineering 93, 286-296 (2006).

Zhao, X.; and Dong, T., Design and fabrication of low-cost 1536-chamber microfluidic microarrays for mood-disorders-related serological studies. Sensors (Basel), 13(11), 14570-14582 (2013).

Zheng, B.; Roach, L.S.; and Ismagilov, R.F., Screening of protein crystallization conditions on a microfluidic chip using nanoliter-size droplets. Journal of the Americal Chemical Society, 125, 11170-11171 (2003). 